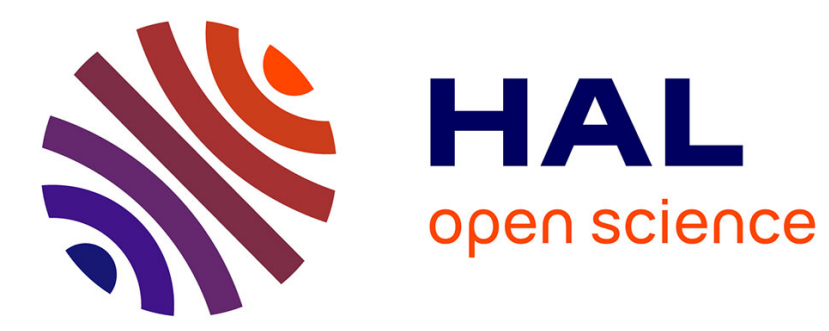

\title{
A Seven-degrees-of-freedom Robot-arm Driven by Pneumatic Artificial Muscles for Humanoid Robots
}

Bertrand Tondu, Serge Ippolito, Jérémie Guiochet, Alain Daidié

\section{To cite this version:}

Bertrand Tondu, Serge Ippolito, Jérémie Guiochet, Alain Daidié. A Seven-degrees-of-freedom Robotarm Driven by Pneumatic Artificial Muscles for Humanoid Robots. The International Journal of Robotics Research, 2005, 24 (4), p.257-274. 10.1177/0278364905052437 . hal-01292939

\section{HAL Id: hal-01292939 \\ https://hal.science/hal-01292939}

Submitted on 24 Mar 2016

HAL is a multi-disciplinary open access archive for the deposit and dissemination of scientific research documents, whether they are published or not. The documents may come from teaching and research institutions in France or abroad, or from public or private research centers.
L'archive ouverte pluridisciplinaire HAL, est destinée au dépôt et à la diffusion de documents scientifiques de niveau recherche, publiés ou non, émanant des établissements d'enseignement et de recherche français ou étrangers, des laboratoires publics ou privés. 


\section{B. Tondu \\ S. Ippolito \\ J. Guiochet}

Laboratoire d'Etude des Systèmes Informatiques et Automatiques

Institut National de Sciences Appliquées,

Campus de Rangueil

31077 Toulouse, France

bertrand.tondu@insa-toulouse.fr

\section{A. Daidie}

Laboratoire de Génie Mécanique de Toulouse

Institut National de Sciences Appliquées,

Campus de Rangueil

31077 Toulouse, France

\section{A Seven-degrees-of- freedom Robot-arm Driven by Pneumatic Artificial Muscles for Humanoid Robots}

robot arms so that they interact with human subjects in total safety. A promising secure way of developing robot-arms adapted to proximity with humans consists of using artificial muscle actuators for which compliance is identical to that of human joints. If chemo-mechanical artificial muscles are still too far removed from practical applications (Hebert, Kant, and De Gennes 1997; Martin and Anderson 1999; BarCohen 2002; Kaneko, Jian Ping Gong, and Osada 2002), pneumatic artificial muscles, notably braided ones, for which the so-called McKibben artificial muscle is the most interesting representative, have proved efficient (Schulte 1961; Matsushita 1968; E.P.W. 1984; Inoue 1988; Caldwell, MedranoCerda, and Goodwin 1995; Tondu and Lopez 1995, 2000; Chou and Hannaford 1996; Caldwell et al. 1999; Davis et al. 2003). Current industrial pneumatic artificial muscles are essentially derived from the McKibben model (Inoue 1968; see Fluidic Muscle MAS, technical document of Festo AG \& Co. KG, Esslingen, Germany; see also The SHADOW Air Muscle, Shadow Robot Group, http://www.shadow.org.uk). With its high power-to-weight and power-to-volume ratios, closely imitating the functions of the natural skeletal muscle, the McKibben muscle is the most adapted artificial muscle for motorizing "soft" and human-size robot arms. Tire manufacturer Bridgestone has shown the possibility of designing and controlling robot-arms entirely actuated by McKibbentype artificial muscles called "rubbertuators" (i.e., rubber actuators) in which revolute joints are driven by two antagonistic muscles. Bridgestone "soft arms" are generally limited to two degrees of freedom (DoF; Noritsugu, Tanaka, and Yamanaha 1996), four DoF (Bridgestone Corporation 1987; Pack, Christopher, and Kawamura 1997) or five DoF (Bridge- 
stone Corporation and Taicubo Engineering 1993). Other robots equipped with McKibben-type artificial muscles are structures limited to a few DoF, such as the ISAC Vanderbilt humanoid robot formed of two 3-DoF arms (Kawamura et al. 1995), the Salford bipedal system composed of two 3 DoF legs and a 2-DoF hip (Caldwell, Medrano-Cerda, and Bowler 1997), or limited to a 1-DoF joint robot in the abstract (Tuijhof and Herder 2000). Our aim is to study the feasibility of designing a 7-DoF anthropomorphic robot-arm motorized by McKibben muscles, within this dual industrial and academic framework. Very recently, the Salford University Robotics team published results concerning the development of a 7-DoF exoskeleton aimed at the rehabilitation and training of an upper limb, motorized by pneumatic McKibben muscles (Tsagarakis and Caldwell 2003). This exoskeleton, which is lighter than $2 \mathrm{~kg}$, has been successful, but resembles more an orthotic device than a robot-arm. On the other hand, it is strictly speaking a 6-DoF structure due to a joint coupling at the shoulder, whereas our 7-DoF anthropomorphic arm ensures true independence between shoulder abductionadduction and flexion-extension motions. In Section 2 of this paper we define the anthropomorphic architecture, in Section 3 we introduce the artificial muscle actuator used, in Section 4 we detail the mechanical design of the robot, and in Section 5 we demonstrate the testing of the teleoperated arm using a pair of joysticks.

\section{Anthropomorphic 7-DoF Robot Kinematic Architecture}

An industrial robot, the tool of which is to be positioned and oriented in space, is typically a six DoF articulated chain. Considering the robot's working area and dexterity, one of the most interesting architectures of the six DoF is the purely revolute architecture associating a 3R carrier known as "revolute type" (Rosheim 1994) or "anthropomorphic type" with a 3R wrist structure essentially of the roll-pitch-roll type. Industrial RX robots derived from classical PUMA robots follow this model. Their wide joint ranges generate a working area that can be globally as wide as that covered by the two arms of a human operator. However, this universal robot has, as a kinematic fault, position singularities specific to the shoulder and elbow, as well as an orientation singularity specific to the wrist (Hollerbach 1985). These singularities reduce the robot's ability when Cartesian trajectories have to be performed. The interest for researchers in robotics of redundant structures arises from the possibility of both solving the question of singularity and avoiding obstacles (KreutzDelgado, Long, and Seraji 1992). Difficulties for controlling redundant robot-arms lead to preferring 7-DoF revolute structures, which are here called 7R robot-arms. One of the 7R's most interesting structures is that derived from the anthropomorphic $6 \mathrm{R}$ structure by adding an elbow roll. The JPL "dexterous arm" (Seraji, Long, and Lee 1993) and the indus- trial Mitsubishi-PA 10 arm were designed according to this structure. The seven DoF of this structure can be described in roll-and-pitch terms by distinguishing three anthropomorphic joint sets performing the following motions: shoulder roll and pitch, elbow roll and pitch, and wrist roll and pitch associated to the tool plate roll.

Furthermore, this kinematic structure can be interpreted as a simple model of the kinematic structure of the human arm, for which the shoulder roll-and-ball socket is modeled by the first three DoF, and the wrist roll-and-ball socket the last three DoF Within the framework of this paper, we propose to apply the terminology of the joint physiology to this anthropomorphic 7R structure rather than the roll-pitch-yaw mechanical terminology. Joint physiology specifies the motions of body links in terms of external-internal rotation, flexion-extension, and abduction-adduction. The use of this terminology implies the choice for a reference body position, called the zeroanatomical position, which is defined as follows. The person stands in an upright position, arms resting along the body, palms turned to the front. In this position, the spatial rotation motions of a mobile link are defined as follows: externalinternal rotation is the rotation of the link along its long axis; flexion-extension is the motion that decreases-increases the angle between jointed links; abduction-adduction moves the link away-towards the body sagittal plane (i.e., dividing the body between its right and its left). Figure 1 illustrates the correspondence which can be established between roll-pitchyaw notation and joint physiology motion notation.

This physiological approach to kinematic robot-arm structures is suggested by the development of humanoid robots in which arms have a first horizontal axis performing flexion-extension or abduction-adduction movements instead of the central vertical axis of industrial robot-arms. For example, the arms of the Honda humanoid robots P2, P3 or Asimo have a 7R structure for which the first axis performs a shoulder flexion-extension (Hirai et al. 1998; http://world.honda.com/ASIMO/). The choice for the first axis - whether shoulder flexion-extension or shoulder abduction-adduction-is free. However, in order to be in agreement with the joint physiology definition of the horizontal flexion-extension (Kapandji 1982), it was decided to consider the shoulder abduction-adduction first. Consequently, if abduction is zero, shoulder flexion-extension is performed on a vertical plane, and on a horizontal plane if abduction is $90^{\circ}$, as illustrated in Figure 2.

Finally, the following sequence was considered for the shoulder: abduction-adduction, flexion-extension, and external-internal rotation. For reasons of symmetry, first abduction-adduction and secondly flexion-extension were also considered for the wrist. Figure 3 shows this 7R structure, where it can be noted that the wrist corresponds in mechanical terms to a roll-pitch-yaw wrist type instead of the roll-pitch-roll wrist type of usual 7R anthropomorphic robotarms. Figure 3(a) specifies the association of the rotation axis 

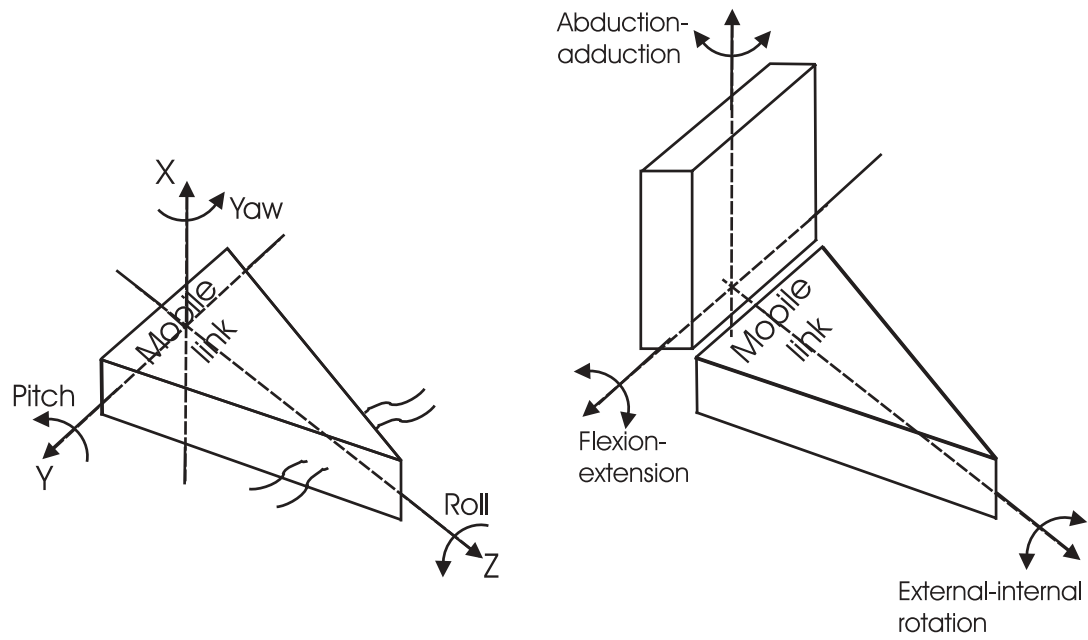

Fig. 1. Correspondence between roll-pitch-yaw mechanical notation and physiological joint motion notation. (a) Classical marine metaphor of roll-pitch-yaw motion of a mobile link seen as a boat sailing along a given reference $Z$-axis. (b) Physiological joint motions of a mobile link jointed to a given link: external-internal rotation corresponds to a roll motion, flexion-extension to a pitch motion and abduction-adduction to a yaw motion (note that the choice for a positive sense is dependent on link physiology).

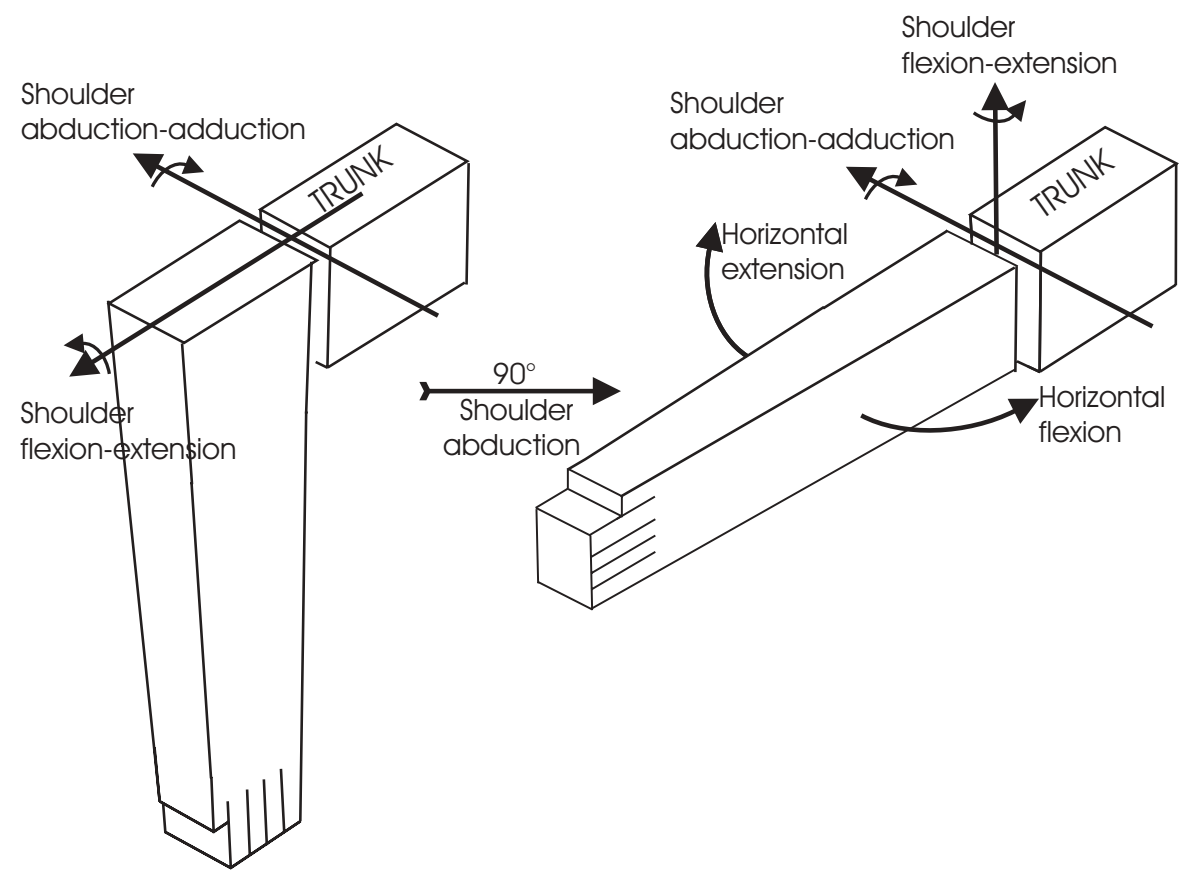

Fig. 2. Argument for considering shoulder abduction-adduction first in the kinematic chain so as to be in agreement with the physiological definition of the shoulder horizontal flexion-extension, as considered in classical joint physiology treatises (Kapandji 1982). 


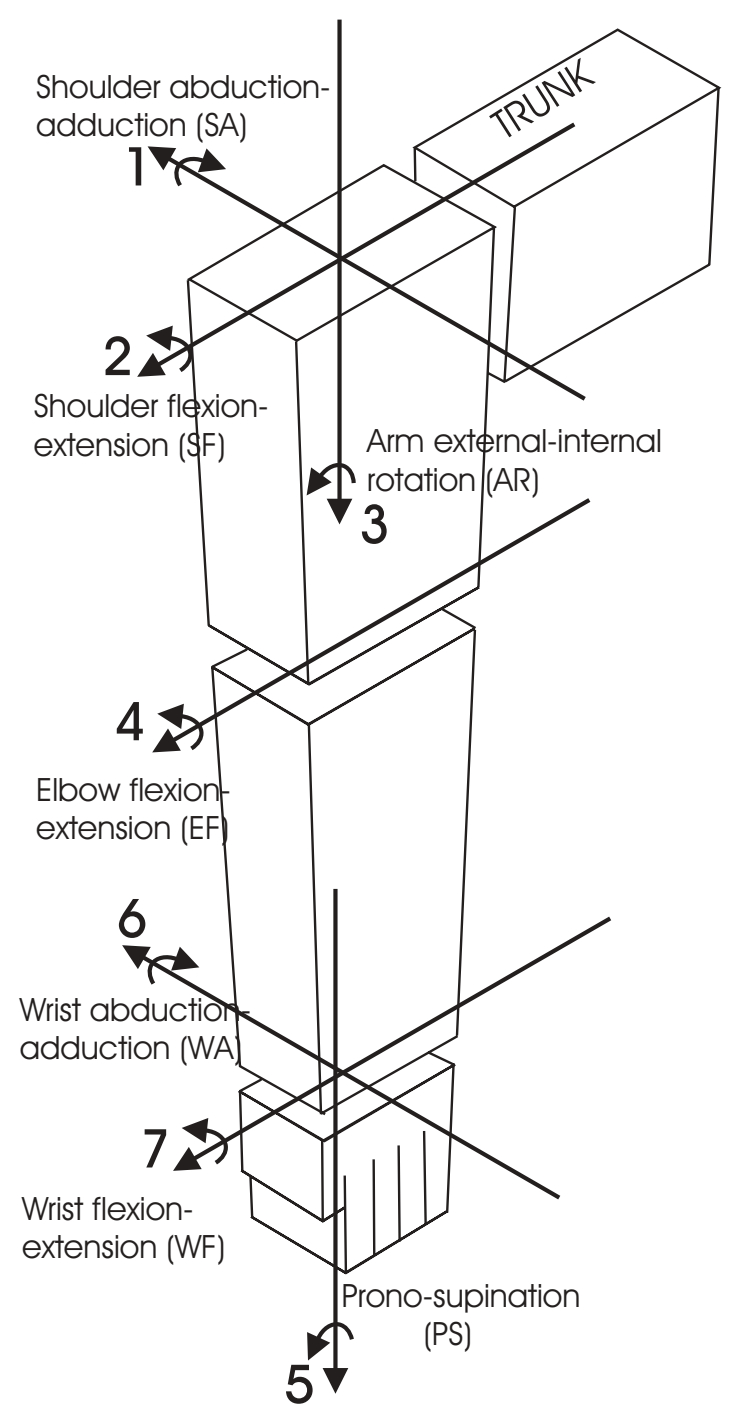

(a)

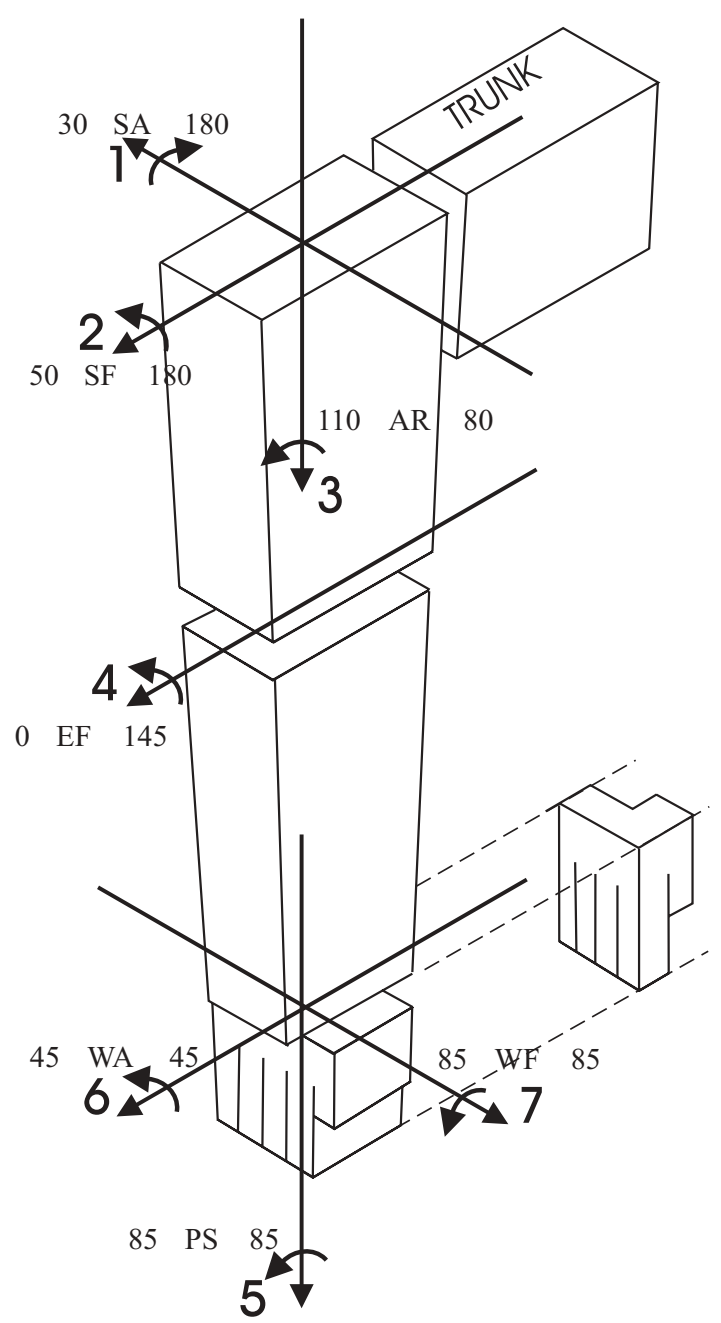

(b)

Fig. 3. Fundamental 7R anthropomorphic structure defined in the joint physiology terminology: (a) zero-anatomical position; (b) considered zero-robot position (indicated joint ranges correspond to anatomical given by Kapandji 1982).

with the bodily structure considered in the form of the three main segments: arm, forearm, and hand, in the so-called zeroanatomical position where the arm falls along the body with the palm open to the front. The positive sense of every joint axis was chosen to correspond to an "opening" motion of the upper limb, as specified in Table 1. Because the wrist is fully stretched in the zero-anatomical position, a zero-robot position is considered in which the wrist joints are in a medium configuration, as illustrated in Figure 3(b). The robot hand in use is a simple two-finger grip mimicking the thumb in opposition to the set of other fingers. Before dealing with the mechanical design of the anthropomorphic robot-arm, the main properties of the McKibben muscle actuators are described, which are to motorize this robot-arm.

\section{Naturally Compliant Artificial Muscle Actuators}

\subsection{Fundamental Model of Artificial Muscle}

Skeletal muscles are characterized by a contraction force depending on the length of the muscle. During physiological contraction, muscle tension decreases in the same way as muscle length. Consequently, the skeletal muscle behaves like a spring for which the stiffness depends on the nervous control of the muscle. Although the nervous system can vary the muscle force in a complex manner, either by recruitment of motor units or modulation of the firing rate of motor neurons (Ghez 1991), the following fundamental linear model of the skeletal 


\begin{tabular}{cccc}
\hline & & & Rotation \\
& Flexion-extension & Abduction-adduction & Pronosupination \\
\hline Positive sense & Flexion & Abduction & External or pronation \\
Negative sense & Extension & Adduction & Internal or supination \\
\hline
\end{tabular}

muscle static force can be considered:

$$
\left\{\begin{array}{c}
F=u f_{\max }\left(1-\frac{\varepsilon}{\varepsilon_{\max }}\right), 0 \leq \varepsilon \leq \varepsilon_{\max } \text { and } 0 \leq u \leq u_{\max } \\
\varepsilon=\left(l_{0}-l\right) / l_{0} .
\end{array}\right.
$$

Here, $u$ designates the value of the nervous control on a $[0$, $\left.u_{\max }\right]$ range, $\varepsilon$ is the contraction ratio of muscle length $l$ with regard to the resting muscle length $l_{0}$ in a $\left[0, \varepsilon_{\max }\right]$ range, and $f_{\max }$ is the maximum force per control unit. From eq. (1), the muscle stiffness equation is obtained:

$$
F_{r}=-u \frac{f_{\max }}{\varepsilon_{\max }} \delta \varepsilon .
$$

This means that the muscle, deviating from its equilibrium position of a $\delta \varepsilon$, returns to this position due to a restoring force $F_{r}$, as illustrated in Figure 4.

This model can be used as a reference model for any type of artificial muscle which is defined as a spring-like device for which the control variable is proportional to its stiffness. A competitive approach defines the notion of artificial muscle from the skeletal muscle in Hill's model, which favors a tension-velocity relationship (Klute, Czerniecki, and Hannaford 1999, 2002). We preferred a tension-length model so as to highlight the "natural compliance" of the artificial muscle actuator resulting from the muscle's spring-like behavior. On the other hand, as opposed to a Hill-based model, using a spring-type model more easily distinguishes the stiffness component from the viscous-type component, which will be considered later. Any artificial muscle must be naturally damped but, especially in the case of fluid artificial muscles, this natural damping depends on the materials used to build the artificial muscle (inner tube, textile braid) as well as the servo-valve supplying the muscle. In particular, the McKibben muscle, as a system where input is the control pressure and output the muscle length, can appear as poorly damped (Klute, Czerniecki, and Hannaford 2002). This means that no damping would occur in response to a physical pressure step. However, in practice, muscle pressure is generated by using a specific pneumatic or hydraulic servo-valve contributing, in conjunction with a judicious choice of textile braid, to naturally damping dynamic muscle behavior (see Figure 5(d)).

\subsection{McKibben Artificial Muscle}

In the fundamental length-tension eq. (1) model, no hypothesis is made on the nature of control variable $u$. The notion of

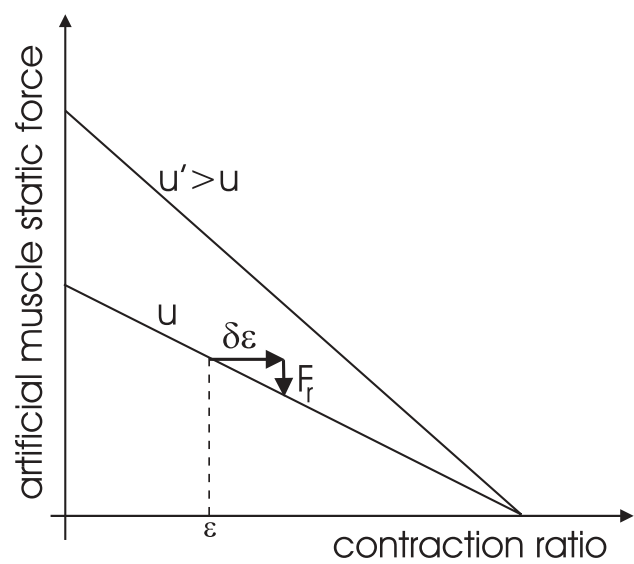

Fig. 4. Linear model of muscle static force production.

the artificial muscle is consequently very free concerning the physical principle that produces the contraction force. However, in previous studies we have shown how beneficial it is to use air-pressurized inner tubes surrounded by braided shell to design artificial muscles adapted to robot-arms. The socalled McKibben artificial muscle today appears as one of the most promising artificial muscles, as proved by the numerous papers devoted to it. Basically, when the McKibben muscle inner tube is under a pressure $P$, the double-helix braided shell surrounding it transforms the circumferential stress of the inner tube into an axial contraction force $F$, which can be expressed in the fundamental generator force model (Tondu and Lopez 2000):

$$
\left\{\begin{array}{l}
F(\varepsilon, P)=\left(\pi r_{0}^{2}\right) P\left[a(1-k \varepsilon)^{2}-b\right], 0 \leq \varepsilon \leq \varepsilon_{\max } \\
\varepsilon=\left(l_{0}-l\right) / l_{0}, a=3 / \tan ^{2}\left(\alpha_{0}\right), b=1 / \sin ^{2}\left(\alpha_{0}\right) .
\end{array}\right.
$$

Instead of contraction ratio $\varepsilon$, an equivalent model considers the current braid angle (i.e., the angle between the muscle axis and one strand of the textile weave) as the muscle characteristic contraction variable (Schulte 1961; Chou and Hannaford 1996; Davis et al. 2003). It seems to us preferable to favor contraction ratio $\varepsilon$ (or the current muscle length) as an output variable in order to more easily derive the antagonistic muscle actuator model. The proposed model characterizes McKibben muscle of current length $l$ by its inner tube, initially cylindrical, of initial length $l_{0}$ and of initial radius $r_{0}$, 
and by its textile weave of initial braid angle $\alpha_{0}$, which is derived from the data of our cotton braid supplier. ${ }^{1}$ Note that the use of cotton gives a natural softness to the braid, which helps to completely cover the inner tube with the braid at rest state. In these conditions, McKibben artificial muscle is clearly geometrically characterized in its non-stretching rest state by the three parameters $l_{0}, r_{0}$, and $\alpha_{0}$. Conversely, the use of plastic-type fibers, as considered by other researchers, creates a braid stiffness which "opens" the braid and makes it stretchable from its rest state to the state in which the braid completely covers the inner tube. There results a real difficulty in equating muscle initial state $l_{0}, r_{0}$ with initial braid angle $\alpha_{0}$ as considered here.

However, the purely cylindrical model with three parameters is too simple to express the artificial muscle behavior as a force generator in a satisfactory manner. This is why we have introduced a correction parameter $k$. Factor $k$ is an empirical factor, experimentally derived, expressing both a bound effect (muscle does not maintain its cylindrical shape at end proximity when it contracts) and the non-integral transmission of pressure force into the muscle weave. Modeling muscle envelope evolution by finite elements, as envisaged by the Washington Biorobotics Laboratory (http://brl.ee.washington.edu), is a promising way to theoretically model the bound effects, but might not be sufficient to fully explain the high dependence between control pressure and real maximum contraction ratio. Faced with this complexity, we preferred to preserve a simple shape of the muscle force generator model by introducing the empirical parameter $k$, which can be estimated as a constant or as a function dependent on pressure $P$.

Compared to the general artificial muscle eq. (1) model, the McKibben muscle tension-length model appears not to be as exactly linear as eq. (3) expresses it. It does however attain the desired artificial muscle nature by generating stiffness globally proportional to the control pressure. We have developed our own artificial McKibben muscles and tested them on the experimental setup, which are used to record muscle parameters during isometric and isotonic contraction as defined by physiology (Ghez 1991). Figure 5(a) shows an isometric contraction for a muscle of $120 \mathrm{~mm}$ initial length, $7 \mathrm{~mm}$ initial radius, and a $23^{\circ}$ initial braid angle, aimed at motorizing the wrist of our 7-DoF prototype. Figure 5(b) shows isometric contraction for a muscle of $230 \mathrm{~mm}$ initial length, $12 \mathrm{~mm}$ initial radius, and a $17^{\circ}$ initial braid angle, aimed at motorizing the robot-arm shoulder. The resulting experimental static tension-length at constant pressure emphasizes the ability of McKibben muscle to develop high maximum force in relation to its own weight and volume. Under 5 bars, the considered muscle can develop a maximum force greater than $5000 \mathrm{~N}$ for

1. The application of textile technology classical formulae indeed gives $\tan \left(\alpha_{0}\right)=\pi\left(D_{i n t}-2 d\right) / p$, where $D_{i n t}$ is the internal diameter of the braid, $d$ is the diameter of the thread, and $p$ is the braid pitch that can be determined from $p=n_{s} / 2 n_{m}$ (in $\mathrm{cm}$ ), where $n_{s}$ is the number of spindles and $n_{m}$ is the number of meshes by centimetre. a weight of about $100 \mathrm{~g}$ and a maximum volume of about 500 $\mathrm{cm}^{3}$. Note that the McKibben muscle maximum force can be dimensioned by imposing the value either of the initial braid angle or of the initial muscle radius. However, as is clearly shown by eq. (3), maximum muscle force is very sensitive to a small variation in the initial braid angle. Consequently, we preferred this parameter to define the set of muscles motorizing the robot, thus obtaining a wide range of maximum contractile force. It is important however to emphasize that the smaller the initial braid angle, the more the muscle radius increases during contraction, which thus requires an adequate choice of rubber able to tolerate the corresponding elongation.

However, the power of the McKibben muscle is limited by its pneumatic nature and particularly by the dynamic behavior of the servo-valve feeding it with pressurized air. The contraction of the McKibben muscle does indeed go with air consumption all the more higher as the initial braid angle is low. Considering a purely cylindrical model of the McKibben muscle (i.e., $r=\left(r_{0} / \sin \left(\alpha_{0}\right)\right) \sqrt{1-\cos ^{2}\left(\alpha_{0}\right)(1-\varepsilon)^{2}}$; Tondu 1995) the following approached expression of the current muscle volume $V(\varepsilon)$ can be derived

$$
V(\varepsilon)=V_{0}(1-\varepsilon)\left(1-\cos ^{2}\left(\alpha_{0}\right)(1-\varepsilon)^{2}\right) / \sin ^{2}\left(\alpha_{0}\right)
$$

where $V_{0}$ is the muscle initial volume $\left(\pi r_{0}^{2} l_{0}\right)$. The result is that the ratio between the current muscle volume and its initial volume has a maximum value corresponding to the theoretical maximum contraction ratio $^{2} \varepsilon_{\max }=1-\left(1 / \sqrt{3} \cos \left(\alpha_{0}\right)\right)$ and equal to $\left(2 / 3 \sqrt{3} \cos \left(\alpha_{0}\right) \sin ^{2}\left(\alpha_{0}\right)\right)$. The classic formula of perfect gases $P V=(m / M) R T$ (where $P$ is pressure, $V$ is volume, $m$ is mass, $M$ is molar mass, $R$ is gas constant, and $T$ is temperature) can be applied to determine air consumption $\Delta m$ between the zero-contraction state and the maximum contraction state for a $\Delta P$ pressure variation. We obtain $\Delta m=\left(2 / 3 \sqrt{3} \cos \alpha_{0} \sin ^{2} \alpha_{0}\right)\left(V_{0} M / R T\right) \Delta P$. In the case of the biggest shoulder muscles of our 7R robot (joint 2: $l_{0}=230 \mathrm{~mm}, r_{0}=12 \mathrm{~mm}, \alpha_{0}=17^{\circ}$ ) the muscle volume initially equal to about $104 \mathrm{~cm}^{3}$ can theoretically increase until about $470 \mathrm{~cm}^{3}$, which for a $\Delta P=5$ bar pressure variation corresponds to an air consumption of $2.85 \mathrm{~g}$, a little less however in practice. In the case of the servo-valves feeding the muscles of our robot-prototype-Samson I/P converters (Samson Corporation, Frankfurt, Electropneumatic Converter $\mathrm{I} / \mathrm{P}$ 5288), generally considered to be rapid in their $0-5$ bar output pressure range - a relatively slow pressure rise results, as shown in the example in Figure 5(c), corresponding to an isotonic contraction of the above-mentioned shoulder muscle picking up a $15 \mathrm{~kg}$ mass in response to a 5 bar numerical step. Corresponding muscle length evolution is given in Figure 5(d). During contraction, maximum muscle power can be estimated at $75 \mathrm{~W}$, which appears as a relatively weak value given the maximum muscle force (in comparison, the skeletal

2. This result is consistent with the fact that the McKibben muscle cannot contract without increasing its volume (Tondu and Lopez 1995). 


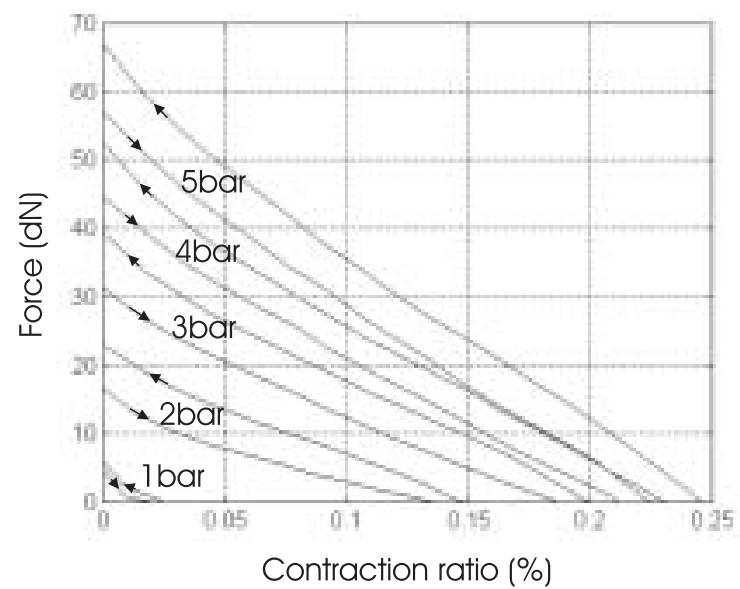

(a)

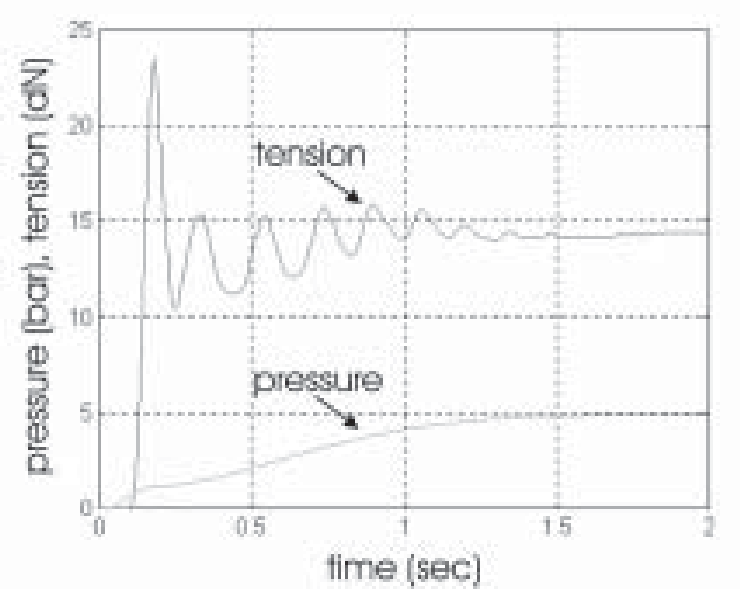

(c)

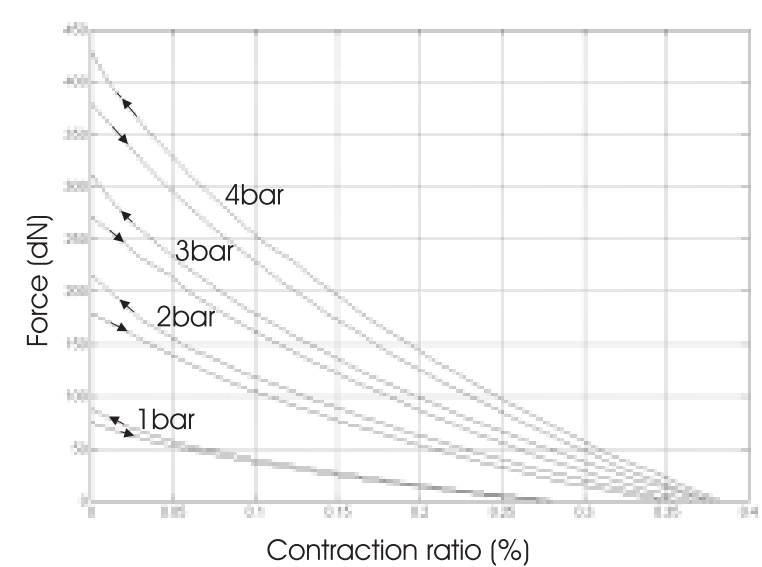

(b)

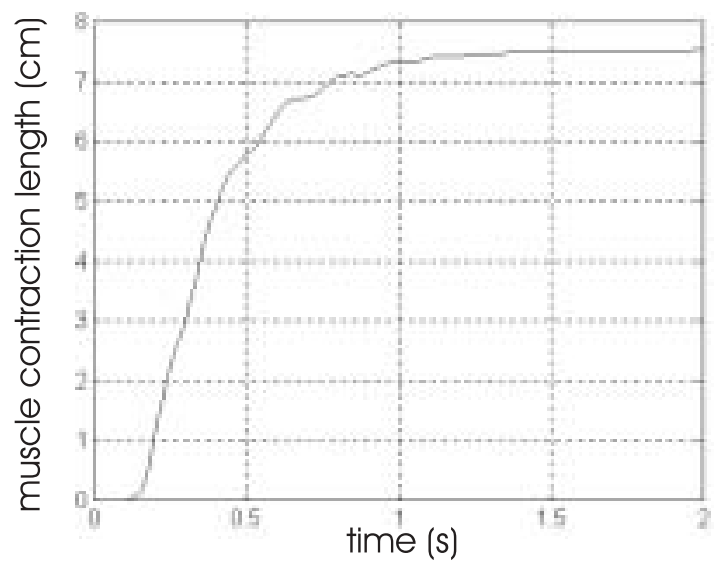

(d)

Fig. 5. Isometric and isotonic recordings of McKibben muscle responses: (a) isometric force response for a 7R robot wrist muscle; (b) isometric force response for a 7R robot shoulder muscle; (c) and (d) isotonic response in pressure, force, and position $x=\left(l_{0}-l\right) / l_{0}$ for the robot shoulder muscle corresponding to (b) isometric response.

muscle develops a maximum power of about $250 \mathrm{~W}$; Bouisset 1987). The increasing of the McKibben muscle power necessitates, in consequence, the increasing of the bandwidth of the servo-valves feeding it. ${ }^{3}$ Interesting proposals have been made with this end in view (Davis et al. 2003). It is important however that the sizes of the valves, just as the sizes of the air pipes, do not become out of proportion to the muscle size.

The low bandwidth of the McKibben muscle corresponds to moderate response times. The McKibben muscle response

3. We have analyzed elsewhere the bandwidth of our I/P converters (Boitier 1996). Its value appears to be independent of the mean working pressure if it is greater than 2 bars but, naturally, depends on output volume; it can be estimated at $5 \mathrm{~Hz}$ for a $75 \mathrm{~cm}^{3}$ volume, at $1 \mathrm{~Hz}$ for a $300 \mathrm{~cm}^{3}$ volume, and at $0.5 \mathrm{~Hz}$ for an $800 \mathrm{~cm}^{3}$ volume. Consequently, the bandwidth of our robot muscles varies as a function of the muscle size between about 1 and $4 \mathrm{~Hz}$. time depends on multiple factors (e.g., initial muscle volume, controlled pressure variation, and load), but is generally between 0.2 and $1 \mathrm{~s}$ (Figure 5(d) gives a typical result for the strongest muscles of our robot which are also the slowest) when the skeletal muscle contracts between 10 and $300 \mathrm{~ms}$ (Boff and Lincoln 1988). This relative slowness of the artificial muscle contraction could appear as a real handicap for the development of robot-arms actuated with McKibben artificial muscles. However, it is important to note that artificial muscle robots aim at performing tasks at "human" speed and accuracy, and today's dynamic McKibben muscle performances are relatively close to those of their human skeletal equivalents. However, researchers have become interested in the abilities of the human arm to perform high-frequency tasks such as drum rolls at a $30 \mathrm{~Hz}$ frequency despite the relative 
slow response of the neuromuscular system (Hajian, Sanchez, and Howe 1997). By testing their hypothesis on McKibben muscles, they have shown how the modulation of muscle passive impedance can explain this paradox. Such an approach could be applied to the control of McKibben artificial muscle robot actuators.

Furthermore, all researchers who have worked on braided muscles have emphasized the difficulty of obtaining an accurate model of muscle contraction (see, for example, Klute 2002). This difficulty is mainly due to the structure itself of the artificial muscle consisting of soft materials whose interactions are complex, and also to the necessity of separating the specific muscle dynamics from the servo-valve dynamics. In a general way, McKibben muscle dynamic force can be written as

$$
F_{d y n}=F-F_{\text {elastic }}-F_{\text {friction }},
$$

where $\mathrm{F}$ is the static force generated by the muscle as expressed in eq. (3), $\mathrm{F}_{\text {elastic }}$ is an elastic force component associated with the inner tube dynamic behavior, and $\mathrm{F}_{\text {friction }}$ is a dry and kinetic complex friction force component associated with the braid dynamic behavior. Friction forces seem to be particularly important in explaining the specific actuator hysteresis (Tondu and Lopez 2000). In comparison with classical actuators, the dynamic complexity of the McKibben muscle appears to be the price to be paid for the natural softness conferred to the robot by the artificial muscle. It implies an increased but non-insuperable control difficulty of the artificial muscle antagonistic actuator.

\subsection{Antagonistic McKibben Artificial Muscle Actuator}

Based on the model of the natural biceps-triceps system, two artificial muscles can be set into antagonism for defining a revolute actuator according to the basic scheme of Figure 6(a). The working principle of the actuator is as follows. The two identical muscles are initially contracted of a ratio $\varepsilon_{0}=\varepsilon_{\max } / 2$ under a pressure of $P_{0}=P_{\max } / 2$, where $P_{\max }$ is generally chosen equal to 5 or 6 bar and $\varepsilon_{\max }$ is generally equal to a value between 0.1 and 0.15 , due to a mean practical maximum contraction range of between 20\% and 30\% (see Figures 5(a) and (b)). From this initial situation, each muscle can contract or lengthen by $\varepsilon_{0} 2$. If the actuator drive is performed by a chain wheel of radius $R$, the motor torque generated by the actuator is given by

$$
T=R\left[F_{1}\left(\varepsilon_{1}, P_{1}\right)-F_{2}\left(\varepsilon_{2}, P_{2}\right)\right] .
$$

In this expression, $F_{1}$ designates the force produced by muscle 1 (defined as that producing positive actuator rotation motion) inflated at pressure $P_{1}$ and contracted of a ratio $\varepsilon_{1}=\varepsilon_{0}-$ $\left(R \theta / l_{0}\right)$, and $F_{2}$ designates the force produced by muscle 2 , inflated at pressure $P_{2}$ and contracted by a ratio $\varepsilon_{2}=\varepsilon_{0}-$ $\left(R \theta / l_{0}\right)$. According to whether pressure $P_{1}>P_{0}$ or $P_{2}>P_{0}$,
$F_{1}$ or $F_{2}$ is an agonistic-type force, the force produced by the other muscle is an antagonistic-type force. From the force generator model of eq. (3), with a $k$ constant typically between 1.25 and 1.35 , the following expression of the static motor torque can be derived:

$$
\left\{\begin{aligned}
T= & K_{1}\left(P_{1}-P_{2}\right)-K_{2}\left(P_{1}+P_{2}\right) \theta, \text { with } \\
& K_{1}=\left(\pi r_{0}^{2}\right) R\left[a\left(1-k \varepsilon_{0}\right)^{2}-b\right] \\
& K_{2}=\left(\pi r_{0}^{2}\right) R 2 a\left(1-k \varepsilon_{0}\right) k R / l_{0} .
\end{aligned}\right.
$$

This model is particularly interesting as it shows how the antagonistic McKibben artificial muscle actuator performs the principle of the equilibrium point found in physiology (Bizzi et al. 1992). According to this principle, the static motor torque can be divided into two effects - an agonistic effect $T_{\text {ago }}$ and an antagonistic effect $T_{\text {antago }}-$ as follows

$$
T=T_{\text {ago }}-T_{\text {antago }}, \text { with }\left\{\begin{array}{c}
T_{\text {ago }}=P_{\text {ago }}\left(K_{1}-K_{2}|\theta|\right) \\
T_{\text {antago }}=P_{\text {antago }}\left(K_{1}+K_{2}|\theta|\right)
\end{array},\right.
$$

where $\left(P_{\text {ago }}, P_{\text {antago }}\right)$ is equal to $\left(P_{1}, P_{2}\right)$ if $\theta \geq 0$ and to $\left(P_{2}\right.$, $\left.P_{1}\right)$ if $\theta \leq 0$.

Equilibrium point $\theta_{\text {equ }}$ is defined by equality $T_{\text {ago }}=T_{\text {antago }}$, which leads to the following expression of $\theta_{\text {equ }}$ :

$$
\theta_{e q u}=\frac{K_{1}\left(P_{1}-P_{2}\right)}{K_{2}\left(P_{1}+P_{2}\right)},
$$

as Figure 6(b) illustrates. When the actuator deviates from the equilibrium point of value $\delta \theta$, it returns to it by means of a restoring torque

$$
T_{r}=-K_{2}\left(P_{1}+P_{2}\right) \delta \theta,
$$

which expresses the actuator stiffness theoretically estimated by the term $K_{2}\left(P_{1}+P_{2}\right)$. Generally speaking, the antagonistic McKibben muscle actuator is a system with a double input $\left(P_{1}\right.$, $P_{2}$ ) and a single output $\theta$, for which the control would need a multivariable approach. However, it is possible to control the actuator as a single input-single output by defining agonistic and antagonistic muscle controls symmetrically as follows: $P_{1}=P_{0}+\Delta P, P_{2}=P_{0}-\Delta P$. Consequently, the static motor torque expression becomes a linear function of the input variable $\Delta P$ and the output variable $\theta$ :

$$
\left\{\begin{array}{l}
T=k_{1} \Delta P-k_{2} \theta, \text { where } \\
k_{1}=2 K_{1}, k_{2}=2 K_{2} P_{0} .
\end{array}\right.
$$

It is important, however, to note that the muscle volume does not change linearly with its contraction ratio. As a consequence, the air consumption of the two antagonistic muscles is not symmetrical. ${ }^{4}$ The result is a slight delay in the buildup

4. From the cylindrical model of the McKibben muscle, it can indeed be derived in accordance with the equations given in Section 3.2 that the muscle volume increases during contraction according to a slope $\mathrm{d} V(\varepsilon) / \mathrm{d} \varepsilon=$ $\left(\pi r_{0}^{2} l_{0}\right)\left[3 \cos ^{2}\left(\alpha_{0}\right)(1-\varepsilon)^{2}-1\right] / \sin ^{2}\left(\alpha_{0}\right)$ positive descending. 


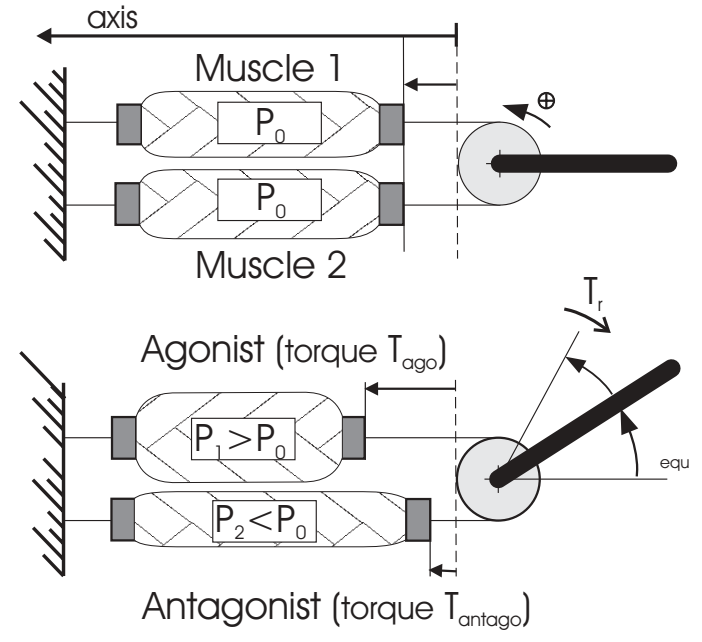

(a)

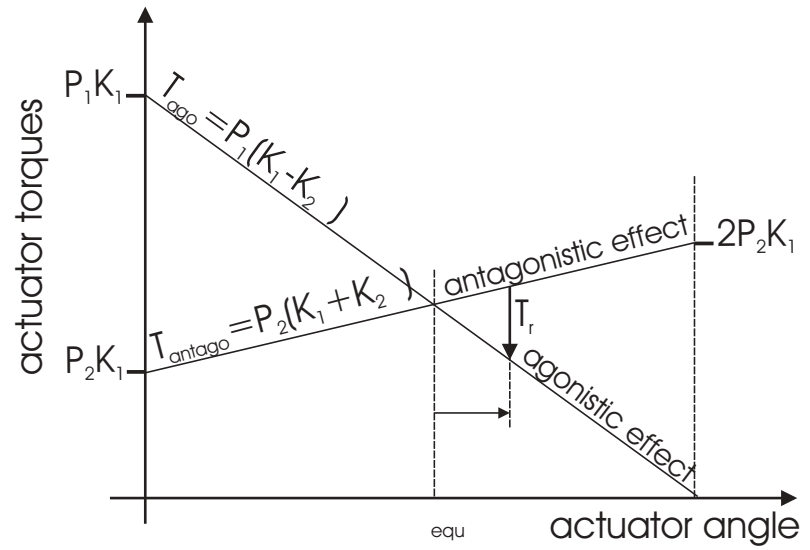

(b)

Fig. 6. Antagonistic McKibben muscle actuator: (a) functioning principle; (b) application of the equilibrium point principle to the antagonistic muscle actuator (illustrated in the case of positive $\theta$ actuator angle).

of the desired pressure in the two antagonistic muscles, all the higher as the two muscles have different contraction ratios, i.e., actuator angle $\theta$ is close to its extreme values.

In the equilibrium position, the actuator behaves like a spiral spring whose stiffness is now independent of $\theta$ and equal to $k_{2}$. This stiffness is particularly adapted to human contact: for example, the elbow flexion-extension joint 4 of our $7 \mathrm{R}$ robot is motorized by muscles of parameters $l_{0}=195 \mathrm{~mm}, r_{0}$ $=8.5 \mathrm{~mm}, \alpha_{0}=19^{\circ}, k=1.25$, with a chain wheel of radius $R=14.5 \mathrm{~mm}$ and $P_{0}=2.5 \mathrm{bar}, \varepsilon_{0}=0.1$, leading to an estimated stiffness of about $15 \mathrm{~N} . \mathrm{m} \cdot \mathrm{rd}^{-1}$ near to values given by joint physiology (Boff and Lincoln 1988). The term "natural compliance" has been given to this joint compliance, which has the same nature as that of the joints of our skeleton. However, this natural compliance would be of no practical use if the actuator were not naturally damped. As previously emphasized, this natural damping of the actuator is due to particularly complex elastic and friction phenomena specific to the muscle components as it is to the servo-valve dynamic behavior. Despite this complexity (and apart from hysteresis behavior) the dynamic expression of the motor torque can be satisfactorily approached by a linear expression as follows

$$
T_{d y n}=a_{1} \Delta P-a_{2} \theta-a_{3} \dot{\theta},
$$

leading to a second-order linear model of the actuator that drives a constant inertial load. Defined in this way, the McKibben artificial muscle actuator is an open-loop stable system. In consequence, the system is easily identifiable and the corresponding identified model can be used as a forward action of a linear or non-linear corrector (Tondu and Lopez 2000).

On the one hand, the antagonistic McKibben artificial muscle actuator offers static performances in maximum force- muscle weight and maximum force-muscle volume ratios well adapted to the motorization of human-size robot-arms. It also offers dynamic performances in response time, damping and compliance relatively well adapted to generating humanlike motions, and, moreover, improvable. However, the maximal contraction that varies between $15 \%$ and $40 \%$, depending on muscle parameters and control pressure, is a major disadvantage of the McKibben muscle. In comparison, the skeletal muscle contracts only $30 \%$ at rest, but in vivo the muscles of vertebrates are stretched by about $10-20 \%$ of their length at rest (Boff and Lincoln 1988). In vivo skeletal muscle can then contract by $40-50 \%$. This structural limitation specific to McKibben muscles imposes, for a desired joint range, the obligation to look for an adequate compromise between muscle length and pulley radius with the possible help of a speed increaser, as will be seen in Section 5. Unfortunately, natural muscle compliance implies that motor torque is highly dependent on the joint variable. An artificial muscle robot is consequently highly dependent on gravity, unlike classical industrial robots, which are free from any gravity effect due to their speed reducers. The design of our anthropomorphic artificial muscle robot-arm aims at taking advantages of the McKibben muscle actuator properties.

\section{Design of the 7R Anthropomorphic Robot-arm}

The classic, and essentially electric, revolute actuators of industrial robotics are characterized as to bulk by a compact structure globally symmetrical around the actuator rotation axis. In comparison, the antagonistic McKibben muscle 
actuator shows very different bulk mechanical characteristics, as follows.

1. First, muscle length is a specific bulk factor because the actuator joint range directly depends on it; this factor is all the more significant as the muscle maximum contraction ratio is limited. Like the human muscularskeletal system, it is possible however to integrate the artificial muscles along the links of the robotized structure.

2. Secondly, the rotation axis is perpendicular to the actuator two-muscle "plane" and located at an edge of the pair of muscles. In consequence, the artificial muscle actuator does not show this axial symmetry peculiar to most revolute actuators.

As a consequence, the design of a robot actuated with artificial muscles must be adapted to the nonclassical character of this revolute actuator, in order to find the right compromise between acceptable dimensions of the robot and desired dynamic performances. The goal of our study consisted of designing a robot-arm where the arm and forearm segments would have lengths similar to those given by human biometry, and the joint ranges are defined according to the 7-DoF model of the human arm presented in Section 2, while minimizing the total weight of the robot. This minimization combined with the joint natural compliance is indeed an essential element for the safe functioning of the robot in a human environment.

\subsection{Dimensioning of the McKibben Artificial Muscle Actuator}

If we consider, as illustrated in Figure 6(a), that each muscle is initially contracted by $\varepsilon_{0}$ the actuator joint range is given by

$$
\theta_{\text {range }}=\left[-\varepsilon_{0} l_{0} / R,+\varepsilon_{0} l_{0} / R\right](\mathrm{rd}) .
$$

Furthermore, in the functioning conditions given in Section 3.3, it appears that the maximum torque in absolute value is generated for a variable control $\Delta P$ equal to $P_{0}$ and for an angular variable $\theta$ equal in absolute value to $\left|\varepsilon_{0} l_{0} / R\right|$. Derived from eq. (10), the following expression of the maximum torque results

$$
T_{\max }=k_{1} P_{0}+k_{2}\left(\varepsilon_{0} l_{0} / R\right),
$$

which can be expressed in the following form by using eq. $(6)^{5}$

$$
T_{\max }=2\left(\pi r_{0}^{2}\right) R\left[a\left(1-k^{2} \varepsilon_{0}^{2}\right)-b\right] P_{0} .
$$

Comparing eqs. (12) and (13), it appears that, if joint range and maximum torque naturally depend both on muscle parameters and driving pulley radius, the joint range does not

5. This result corrects the incomplete analysis of maximum torque generated by the McKibben muscle actuator we have previously developed (Tondu and Lopez 2000). depend on $\alpha_{0}$ and $r_{0}$ Consequently, these latter two parameters can be specifically used for the dimensioning of the maximum torque. It is also important to note that the joint range directly depends on initial muscle length $l_{0}$, but a lengthening of the muscles unfortunately increases the bulkiness of corresponding mechanical parts supporting the muscles. If pulley radius $R$ plays the classic role of increasing or decreasing joint range and maximum torque in an inverse ratio, initial muscle contraction $\varepsilon_{0}$ plays a specific role this study would like to emphasize. Its value has a weak influence on the maximum torque and a large influence on the joint range. As previously stated, a typical range for $\varepsilon_{0}$ is $0.1-0.15$ but it can be interesting to adjust this value accurately, either to increase the maximum joint range by choosing $\varepsilon_{0}$ near the estimated maximum value, or to use $\varepsilon_{0}$ as a factor for tuning the corresponding joint mechanical stop. This approach will be used in our robot prototype.

Furthermore, the current actuator torque appears to depend on the angular variable generated by the actuator, in analogy with human gestures. For the robot actuated with artificial muscles, a load manipulation results fundamentally different from that of industrial robots, which is generally performed at constant torque. The consequence of this joint dependence of the actuator torque will be analyzed in Section 5 when the robot moves against gravity.

\subsection{Mechanical Arm Design Based on the Use of CAD Modeling ${ }^{6}$}

Mechanical arm design aims to develop technological solutions adapted to the artificial muscle actuator, which combines arm compactness globally equivalent to that of the human arm, with joint ranges approaching those of the human arm, as indicated in Figure 3(b).

In comparison with Bridgestone soft-arms and derived robots, the relatively advanced anthropomorphic character of our robot-arm has led us to develop original solutions for the shoulder and the wrist design in order to achieve specific abduction-adduction and flexion-extension motions. The $\theta_{1}$ and $\theta_{2}$ shoulder joints impose the most difficult muscle dimension constraints because the corresponding joint actuators have to develop the highest motor torques for driving the arm against gravity. Making these two joints independent is also a major difficulty, as already emphasized by Tsagarakis and Caldwell (2003) whose McKibben muscle exoskeleton shows a shoulder flexion-extension and abduction-adduction dependence. The original solution we have considered to obtain this independence is based on the possibility of contracting the two ends of the McKibben muscle simultaneously. Figure 7 illustrates the joint 1 and joint 2 corresponding mechanical system. The joint 1 muscle pair is fixed to the robot frame. It

6. Details of the mechanical design developed on I-DEA CAD software are not given within the framework of this paper. A more specifically mechanical paper presenting the design of our anthropomorphic arm is being prepared. 


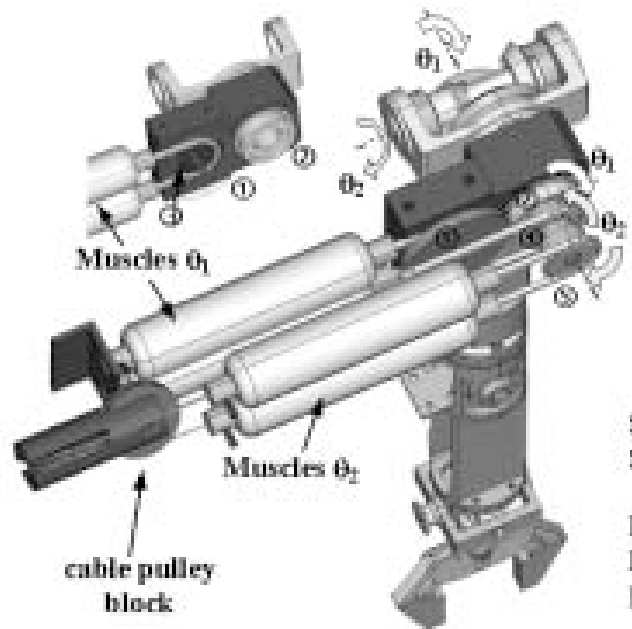

(a)

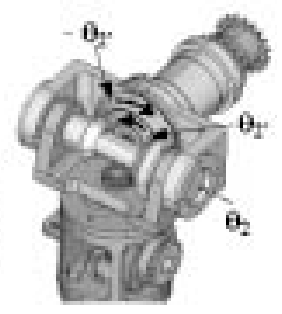

Spur gear: (1)

Spur zear: 0

Roller chain: G

Roller chain: 6

Roller chain: 8

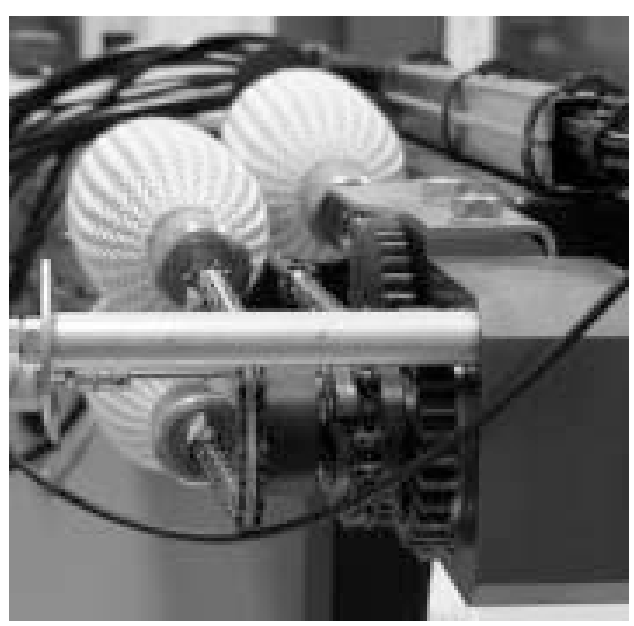

(b)

Fig. 7. Mechanical design of the robot shoulder: (a) general diagram illustrating the independence principle for joint 1 and joint 2 motions; (b) photograph of the two muscle pairs driving the robot shoulder joints.

controls a pair of spur gears $(\varphi$ and $\kappa)$, driven by the actuator pulley $\lambda$, which amplifies the actuator angular motion in a 1.5 ratio. In opposition, joint 2 muscles can contract their two ends simultaneously. Joint 2 moves according to a differential principle performed by two bevel gears, functioning in opposition by means of a back pulley mechanism. When joint 2 muscles are controlled, they both act on pulleys $\mu$ and $v$ generating corresponding opposite angular displacements $\left(-\theta_{2}^{\prime}\right.$ and $\left.+\theta_{2}^{\prime}\right)$. Consequently, joint 2 can move independently of joint 1 , and when joint 2 does not move, the joint 2 driving system acts as an extensible tie that prevents pulleys $\mu$ and $v$ from rotating. As in the case of joint 1 , the joint 2 bevel gear system amplifies the actuator angular motion in a 1.5 ratio. It is however important to note that if the considered mechanical system of joint 2 can generate a torque equivalent to that which would be obtained with a fixed end, the corresponding joint range is divided by 2 since it corresponds to twice the muscle contraction when one end is fixed. This joint range deficiency could be compensated by doubling the muscle length but would lead to unduly increasing the robot frame length. Consequently, joint 2 muscles are very slightly oversized compared to joint 1 muscles with a corresponding $\varepsilon_{0}$ tuned to its maximum value. As mentioned in Table 2, we can therefore hope to expect a $180^{\circ}$ joint 1 range and a $100^{\circ}$ joint 2 range with maximum torques greater than $60 \mathrm{~N} \mathrm{~m}$.

Wrist flexion-extension and abduction-adduction are not so severely constrained in torque and joint range performances. Joint 6 is directly driven by its muscle pair as illustrated in Figure 8(a), and joint 7 is independently directly driven by means of a swing-bar of half length $L_{7}$ size which plays the role of the pulley radius $R$ of other joint actuators, as illustrated in Figures 8(b) and (c). This mechanical solution gives the wrist a compactness in accordance with the desired human-like robot dimensions.

Table 2 synthesizes the set of muscles and transmission system characteristics. They were selected so as to generate anatomically desired joint ranges with associated maximum possible torque, while maintaining robot-arm dimensions similar to those of human arms. Note that the given value of "peak force" can be estimated from torque eq. (13) (with a typical $k$ equal to 1.25) divided by the ratio $m_{V}$. Figure 9 gives the resulting prototype. Its arm, in the anatomical sense, has a $351 \mathrm{~mm}$ length between axes, and its forearm a $307 \mathrm{~mm}$ length between axes. The lateral frame supporting the shoulder muscles is $350 \mathrm{~mm}$ wide. The hand is a simple two-finger grip actuated by a double-effect pneumatic cylinder. Figure 10 shows the current development of the robot with its muscle actuators. The total weight of the robot's mobile parts including muscles and chains is about $6 \mathrm{~kg}$ and the robot's total weight with its shoulder (fixed base) is about $10 \mathrm{~kg}$. The weight of the pneumatic interface, which is essentially the weight of the $14 \mathrm{I} / \mathrm{P}$ converters supplying the muscles as the I/P converter supplying the robot tool-plate, is about $10 \mathrm{~kg}$. These converters have been set in a lateral box attached to the robot frame. The size of used I/P converters is a bulk factor (each $\mathrm{I} / \mathrm{P}$ converter has a volume more or less equal to $500 \mathrm{~cm}^{3}$ ) but commercialized smaller I/P converters could be expected in the future. The supply requirement is essentially pneumatic; the electrical supply requirement is limited to the supply of the I/P converters, sensors and a robot controller. The robot pneumatic supply of the arm is ensured by the University's air-supply system ( 6 bar). This supply could be attached to the robot under the form of a high-pressure gas tank, although the energy range of the robot is limited by the high air consump- 


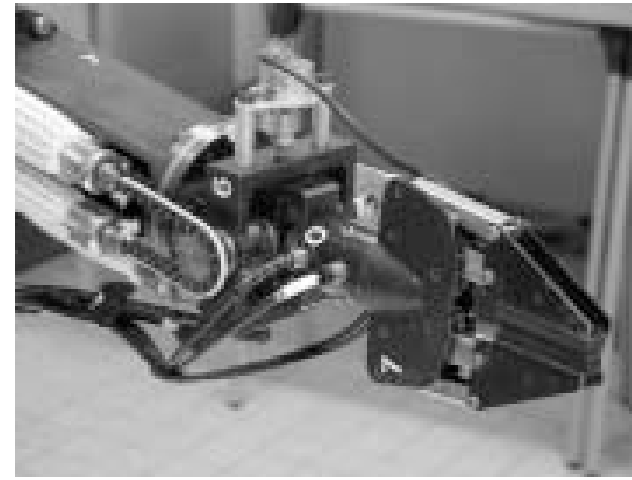

(a)

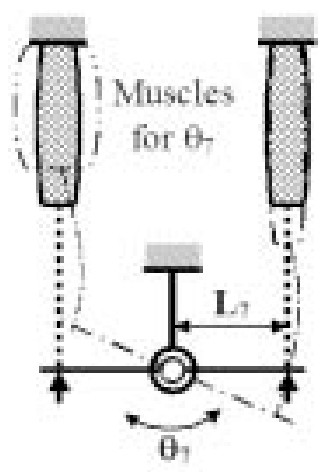

(b)

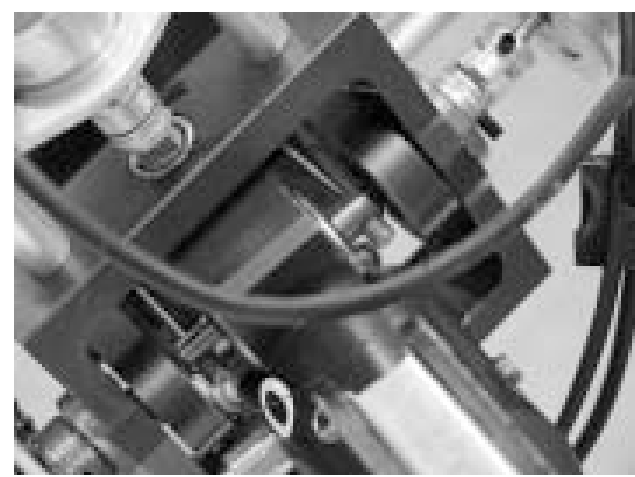

(c)

Fig. 8. Mechanical design of the robot wrist: (a) photograph of the robot wrist showing joint 6 direct drive; (b) diagram of joint 7 transmission by swing-bar; (c) photograph of joint 7 transmission.

Table 2. Actuators Characteristics

\begin{tabular}{|c|c|c|c|c|c|c|c|c|c|}
\hline \multirow[b]{2}{*}{$\begin{array}{l}\text { Joint } \\
\text { Number }\end{array}$} & \multicolumn{3}{|c|}{$\begin{array}{c}\text { Muscle } \\
\text { Characteristics }\end{array}$} & \multicolumn{2}{|c|}{$\begin{array}{l}\text { Pulley-Chain } \\
\text { System } \\
\text { Characteristics }\end{array}$} & \multicolumn{2}{|c|}{$\begin{array}{l}\text { Transmission } \\
\text { System } \\
\text { Characteristics }\end{array}$} & \multirow{2}{*}{$\begin{array}{c}\text { Estimated } \\
\text { Joint Range } \\
(\mathrm{deg})\end{array}$} & \multirow{2}{*}{$\begin{array}{c}\text { Estimated } \\
\text { Peak } \\
\text { Torque } \\
\text { (N.m) }\end{array}$} \\
\hline & $\begin{array}{c}l_{0} \\
(\mathrm{~mm})\end{array}$ & $\begin{array}{c}r_{0} \\
(\mathrm{~mm})\end{array}$ & $\begin{array}{c}\alpha_{0} \\
(\mathrm{deg})\end{array}$ & $\varepsilon_{0}$ & $\begin{array}{c}R \\
(\mathrm{~mm}) \\
\end{array}$ & Mode & $m_{v}$ & & \\
\hline 1 & 195 & 12 & 17 & 0.11 & 20.5 & Cylindrical gear & 1.5 & {$\left[0^{\circ}-180^{\circ}\right]$} & 61 \\
\hline 2 & 230 & 12 & 17 & 0.15 & 23 & $\begin{array}{l}\text { Double bevel } \\
\text { gear in } \\
\text { opposition }\end{array}$ & 1.5 & {$\left[0^{\circ}-100^{\circ}\right]$} & 67 \\
\hline 3 & 140 & 8.5 & 20 & 0.15 & 18 & Bevel gear & 1.5 & {$\left[-100^{\circ},+100^{\circ}\right]$} & 18 \\
\hline 4 & 195 & 8.5 & 19 & 0.10 & 14.5 & Direct drive & 1 & {$\left[0^{\circ},+150^{\circ}\right]$} & 25 \\
\hline 5 & 120 & 7 & 23 & 0.08 & 12.5 & Bevel gear & 2 & {$\left[-90^{\circ},+90^{\circ}\right]$} & 5 \\
\hline 6 & 120 & 7 & 23 & 0.07 & 12.5 & Direct drive & 1 & {$\left[-35^{\circ},+35\right]$} & 10 \\
\hline 7 & 120 & 7 & 23 & 0.11 & 17.5 & $\begin{array}{l}\text { Direct drive by } \\
\text { cross-bar }\end{array}$ & 1 & {$\left[-45^{\circ},+45^{\circ}\right]$} & 13 \\
\hline
\end{tabular}

tion of McKibben muscles, as mentioned earlier. However, the latter could be reduced by a pre-filling of the artificial muscle (Davis et al. 2003) or by a reprocessing of the pressurized air between the two actuator muscles.

\section{Experimental Validation of the Prototype}

The robot was tested in a teleoperation mode by means of two three-axis joysticks that control joints 1, 2, 4 and joints 5, 6, 7 , and a swing bar controlling joint 3 . Joint position can be controlled in open loop since the antagonistic muscle actuator is stable in open loop or in closed loop through a simple linear PID controller; the problem of actuator control is not discussed in this paper, as it was dealt with in our extensive paper (Tondu and Lopez 2000) and discussed elsewhere (Hesselroth et al. 1994; Van der Smagt, Groen, and Schulten 1996;
Cai and Yamaura 1997). The teleoperation of the robot has a double goal: testing real joint ranges especially against gravity, which is a fundamental character for humanoid robotics, and testing effective robot compliance when tasks involving a contact of the robot with its environment are performed.

\subsection{Test of Robot Joint Ranges Against Gravity}

It is well known that the joints of a robot-manipulator are influenced by dynamic effects from other joints and from the static effect of gravity. In classical industrial robots, these effects are generally diminished by using high ratio speed reducers associated with high-speed rotating actuators. Such a strategy is impossible in the case of our robot because the natural compliance of the artificial muscle will be lost. Direct driving or driving by angle multiplier of moderate ratio are 


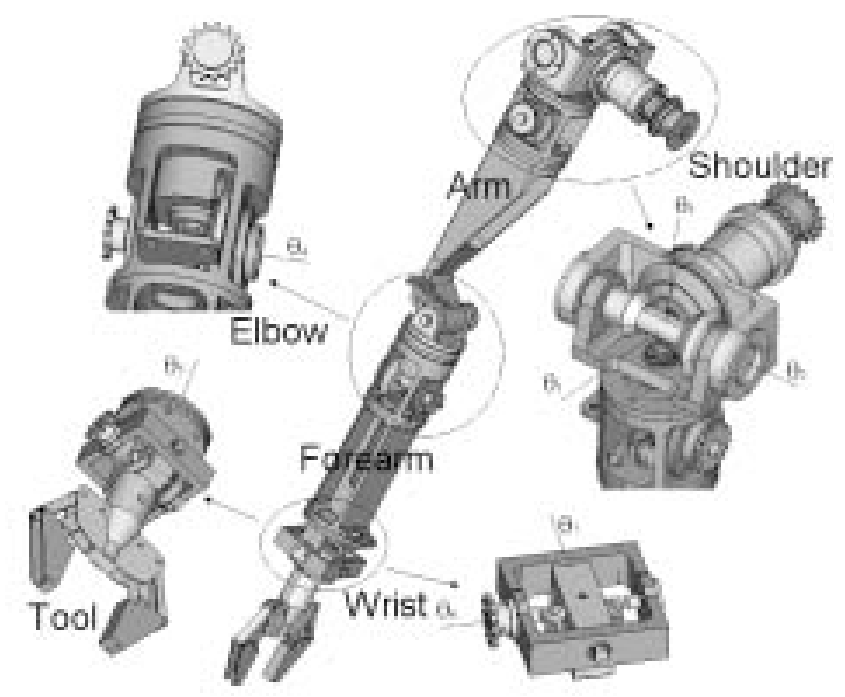

Fig. 9. General diagram of the main parts of the anthropomorphic robot-arm mechanical design.

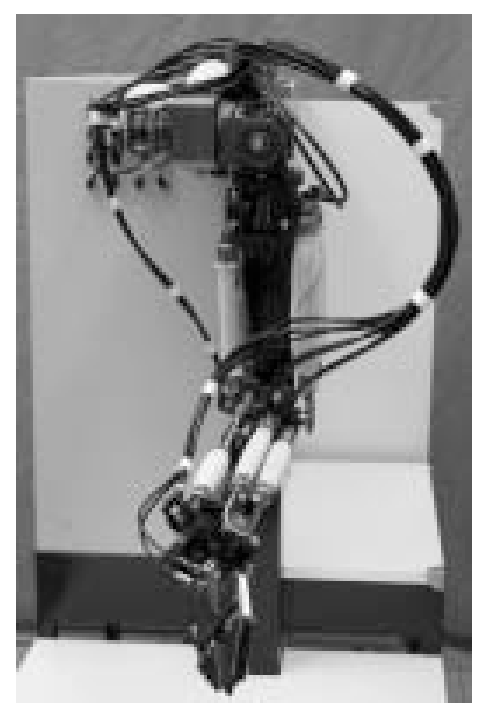

(a)

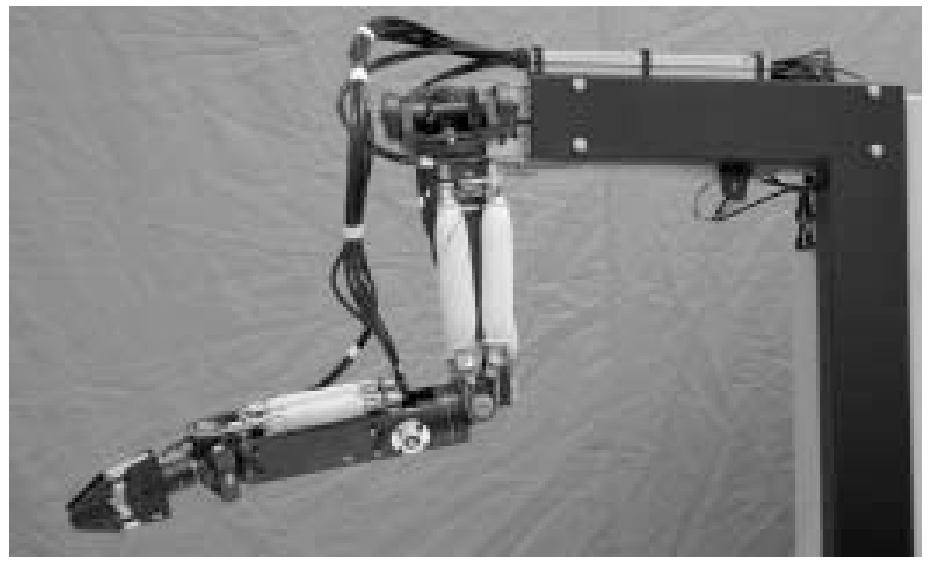

(b)

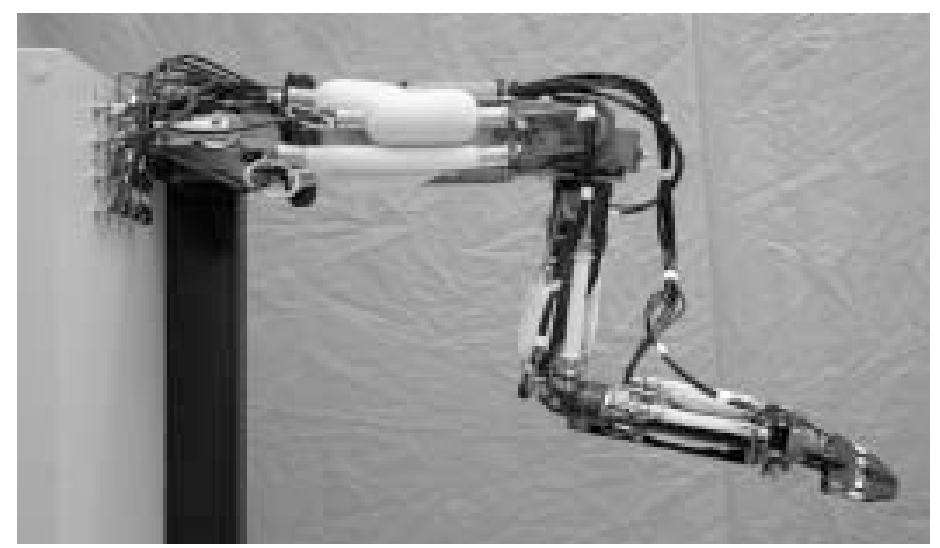

(c)

Fig. 10. Photographs of the $7 R$ anthropomorphic arm: (a) lateral view photograph; (b) front view photograph; (c) rear view photograph. 
the only adapted driving modes. The first consequence is the dynamic non-linear behavior of the artificial muscle actuator, which must be taken into account by the robot (Tondu and Lopez 2000). It must also be noted, however, that the field of applications to be performed by this kind of soft robot-arm necessitates generally moderate speeds and accelerations. The second consequence that we wish to study here is the static behavior of robot against gravity. This behavior is submitted to the dependence of the actuator and gravity torques on actuator angle, as Figure 11 illustrates. Using the actuator static model of eq. (10), including a speed increaser of ratio $m_{V}$, and notations of Figure 11(a), the following equation expressing the total joint torque submitted to the gravity effect is obtained:

$$
T_{\text {joint }}=\left(1 / m_{V}\right) k_{1} \Delta P-\left(1 / m_{V}\right)^{2} k_{2} \theta_{\text {joint }}-m g l \cos \theta_{\text {joint }} .
$$

A typical simulation of joint eq. (14) in a $\left[-90^{\circ},+90^{\circ}\right]$ angular range is given in Figure 11(b) assuming a $\Delta P$ constant equal to the maximum value $\left(P_{0} / 2\right)$. It highlights the fact that the total torque can take a minimum value for a joint angle between $0^{\circ}$ and $90^{\circ}$. If this minimum value is negative, due, for example, to a load at the robot end-effector, the maximum corresponding joint angle will be equal to the joint value corresponding to a total torque equal to zero. The flexion-extension and abduction-adduction shoulder joints are particularly sensitive to this situation because their actuator could have to face maximum resistive gravity torque when the forearm is in full extension. When a large joint range is desired, associated with the need for high torques, as required by the shoulder joints, it is possible to associate power muscles with a speed increaser of moderate ratio. Figure 11(c) gives the simulation result obtained with the joint 1 parameters of the $7 \mathrm{R}$ robot moving in full forearm extension. Without any load, it appears clearly that the full $180^{\circ}$ desired joint range is obtained. This is also the case if the robot tool carries a $1 \mathrm{~kg}$ load. However, if the end-effector robot load becomes equal to $2 \mathrm{~kg}$, the effective joint range is limited to about $90^{\circ}$ due to the typical joint response against gravity. Such simulation illustrates a typical property of robot limbs actuated with artificial muscles: joint ranges are highly gravity-dependent.

Figure 12 shows the variations in movement of the seven joints of the robot controlled at very slow speed to eliminate dynamic effects. Joint 1 and joint 2 motions were recorded at full-arm extension. The theoretical estimated full angular range was checked for every joint and the effect of carrying loads tested. By comparison with anatomical values, all joint ranges can be performed by our anthropomorphic robot-arm except for joint 2 , for which current range at full forearm extension is limited to about $\left[0^{\circ}, 100^{\circ}\right]$. Such a phenomenon highlights the difficulty of designing compact and powerful shoulders performing both flexion-extension and abductionadduction. For other mechanical reasons, Honda humanoid P3 is unable to lift its extended arms higher than the horizontal and robot Asimo limits its maximum shoulder "joint mount- ing" to about $110^{\circ}$, due to the overlapping of the two shoulder freedoms of movement, as mentioned in the available technical data (http://world.honda.com/ASIMO/). The paradoxical difficulty of lifting a $6 \mathrm{~kg}$ anthropomorphic robot-arm actuated with artificial shoulder artificial muscles generating a maximum force close to $5000 \mathrm{~N}$ highlights a double specificity of human body mobility. First, human body links are relatively light since the upper limb weighs only about $3.6 \mathrm{~kg}$ (Winter 1969). Only the use of composite materials would result in our robot-arm prototype being lightened to this anatomical value. Secondly, the control of natural motions is based on muscular synergies. Let us recall that the human shoulder is governed by about 10 main muscles, for which the control sequence depends on the arm elevation angle.

\subsection{Soft Task Test}

To test its ability to perform soft grasping manipulation tasks, we teleoperated the robot-arm in joint space conditions to grasp a glass and put it back on to a hard surface, as Figure 13 illustrates. When the glass is on the table it is still possible, even in joint space control condition, to make it slide on the table without breaking it. Such an academic task stresses the surprising skills of artificial muscle actuators to imitate the human touch.

The compliance of the robot-tip is not controlled in this experiment but it is important to note that it is possible to define a stiffness matrix in the Cartesian space. If we note $\mathbf{q}$ as the vector of the robot joint variables, $\mathbf{T}_{r}$ the vector of joint restoring torques, $\mathbf{x}$ the robot-tip location vector and $\mathbf{F}_{r}$ the corresponding restoring force-torque vector in the Cartesian space, the application of the virtual works theorem gives

$$
\delta \mathbf{x}^{\mathbf{T}} \cdot \mathbf{F}_{\mathbf{r}}=\delta \mathbf{q}^{\mathbf{T}} \cdot \mathbf{T}_{\mathbf{r}}
$$

By considering the Jacobian matrix of the robot $\mathbf{J}$ and its pseudo-inverse $\mathbf{J}^{+}$, we could write

$$
\begin{aligned}
\mathbf{F}_{\mathbf{r}} & =-\mathbf{K} \delta \mathbf{x} \\
\text { with } \mathbf{K} & =\left(\mathbf{J}^{+}\right)^{T} \mathbf{K}_{\mathbf{q}} \mathbf{J}^{+}
\end{aligned}
$$

where $\mathbf{K}_{q}$ represents the joint stiffness matrix and $\mathbf{K}$ is the corresponding Cartesian stiffness matrix. $\mathbf{K}_{q}$ is a diagonal matrix, the terms of which have, from eq. (9), the general form $K_{2}\left(P_{1}+P_{2}\right)$ reducing to $k_{2}$ in case of symmetrical pressure control, if necessary multiplied by a transmission term such as $\left(1 / m_{V}\right)^{2}$. In the framework of our paper, eq. (15) expresses a simple fact: if we motorize a robot-arm kinematically analogous to the human arm with actuators whose stiffnesses are similar to those of corresponding human joints, the compliance at the tip of the robot-arm will be similar to that of our arm. Further experiments will aim to quantify the ability of such 7R anthropomorphic robot-arms actuated with artificial muscles, to reproduce natural human touch in world space. 


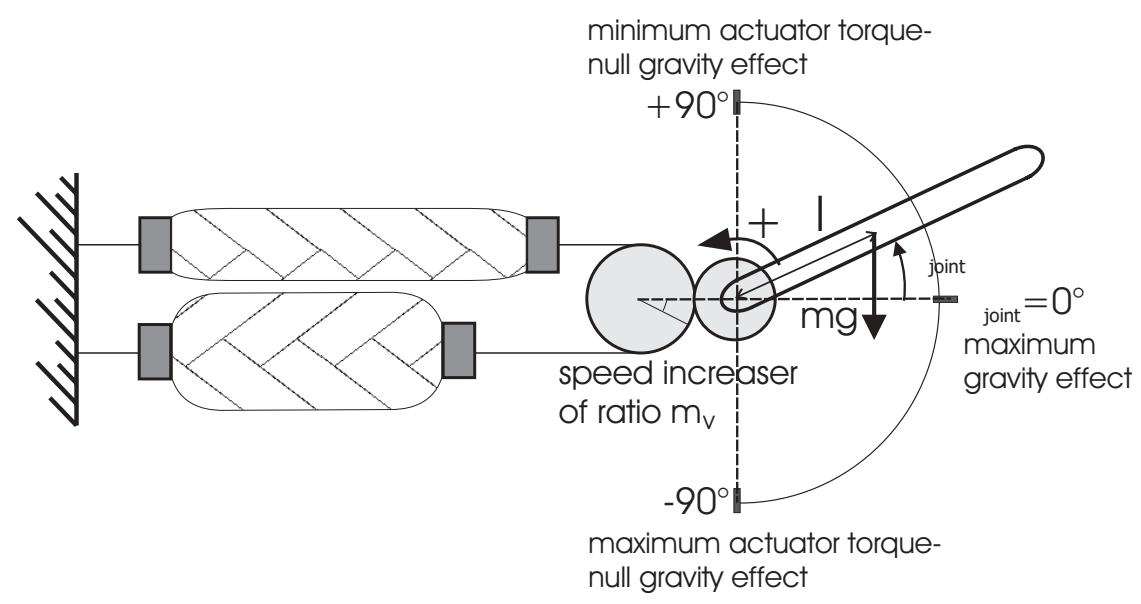

(a)

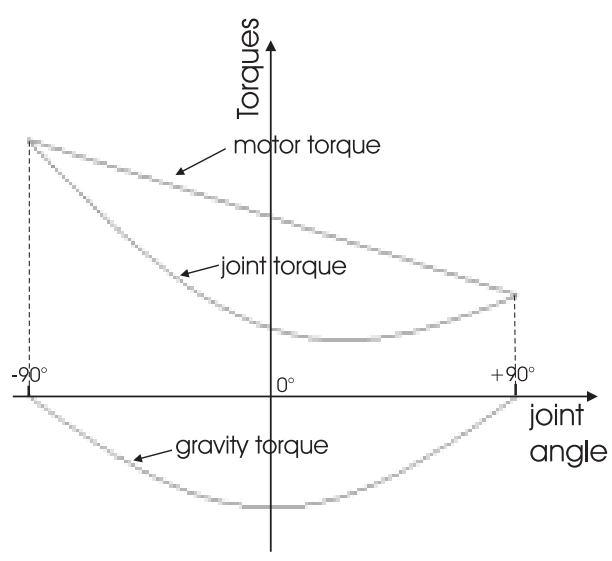

(b)

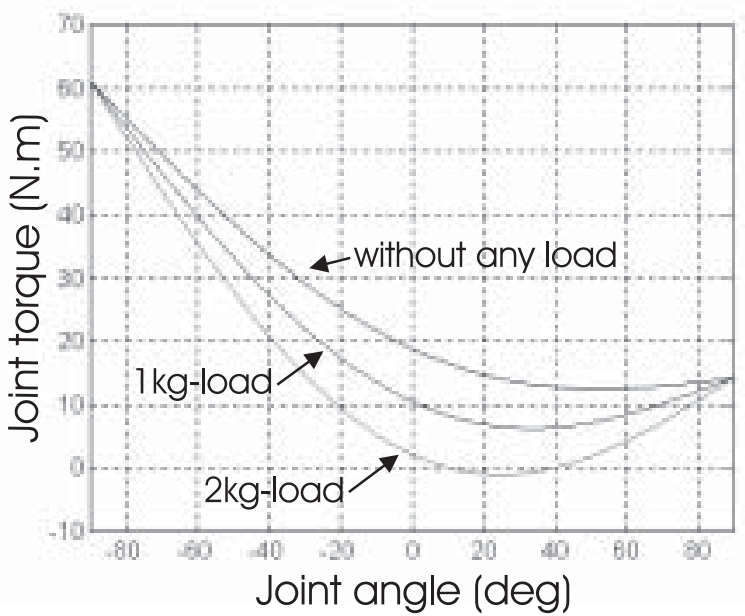

(c)

Fig. 11. Theoretical analysis of the behavior of the antagonistic artificial muscle actuator behavior against gravity: (a) notations; (b) dependence of the motor torque and the gravity torque on the joint angle; (c) simulation of the joint torque according to the load carried by the robot tool.

\section{Conclusion}

McKibben artificial muscles offer large possibilities for the actuation of human-friendly artificial limbs. The pneumatic actuator, defined by two identical antagonistic McKibben muscles driving a revolute axis, presents very interesting power-to-weight and power-to-volume ratios in comparison with other fluid or chemical artificial muscles. It also presents a true analogy with a skeletal muscle in generating a naturally damped contraction force decreasing with the muscle length. The consequence is a natural joint compliance, which generates the "human touch" of the robot. However, the soft materials (rubber and textile fibers) used in the muscle de- sign are a complicating factor leading to force-length characteristics difficult to model, and presenting a hysteresis phenomenon. The maximum contraction ratio is a real disadvantage for generating a wide joint range. Our research has aimed to test the validity of designing a robot-arm actuated by pneumatic artificial muscles, containing a human-like size forearm and arm with joint ranges approaching elementary physiological joint motion ranges in abduction-adduction, flexionextension, and external-internal rotation. Our 7R prototype motorized by antagonistic McKibben artificial muscles has validated this possibility; it fits into the development of soft arms, as produced by Bridgestone, the Japanese tire manufacturers, leaders in the field in the 1980s and 1990s. The 

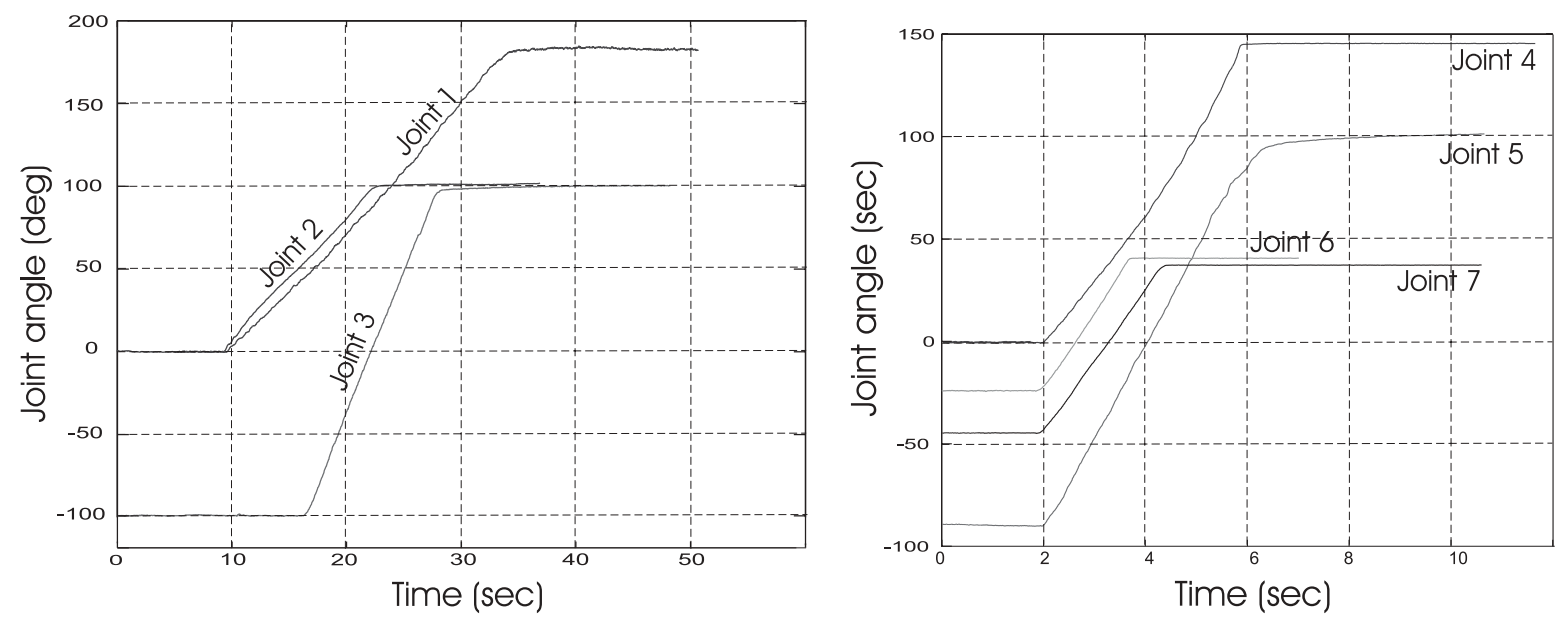

Fig. 12. Experimental recording of the 7R anthropomorphic joint evolutions (joint zero values correspond to the Figure 3(b) zero-robot position): (a) joint 1-3 motions performed with the arm fully extended; (b) joint 4-7 motions.
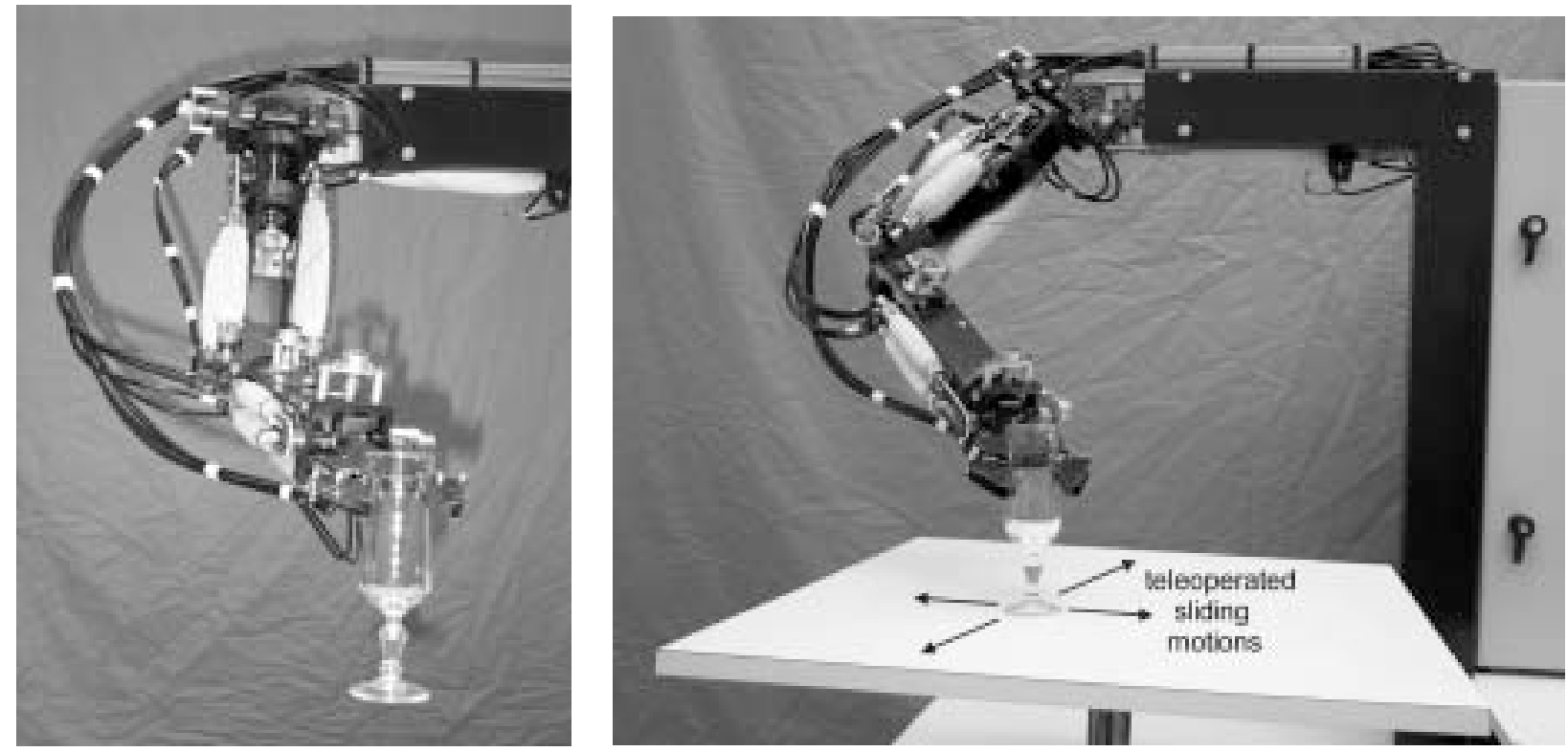

Fig. 13. Picking up and placing: the teleoperated task of a fragile object, highlighting both actuator sensitivity and its natural compliance.

"human touch" of our 7R anthropomorphic robot arm has been highlighted by controlling it by teleoperation. However, the natural joint compliance, a direct consequence of the springlike functioning of artificial muscles, implies a dependence of the actuator torque with joint angle, particularly important for shoulder control. It implies that the robot-arm is sensitive to gravity. In particular, the elevation or adduction of the robot-arm shows a particular point at which the joint torque becomes minimal against gravity. A negative value of this particular point limits the joint range, especially when the robot lifts a load. Although joint 1 and joint 2 muscles of our robot can develop a particularly high maximum force, shoulder elevation is limited in comparison with human shoulder possibilities. Better shoulder motion abilities could be obtained by further anthropomorphism. It is known that the human arm is controlled by more than 50 muscles in a complex synergy. The use of artificial muscles such as a robot actuator of a human-like robot would lead to the development of a true artificial musculature, controlling a model of the shoulder complex as the Washington Biorobotics Laboratory 
(http://brl.ee.washington.edu) anthroform project has tried to initiate (Hannaford et al. 1995).

\section{Acknowledgments}

We would like to express our sincere thanks to the anonymous reviewers for their very pertinent remarks.

\section{References}

Bar-Cohen, Y. 2002. Electroactive polymers as artificial muscles: a review. Journal of Spacecraft and Rockets 39(6):822-827.

Bizzi, E., Hogan, N., Mussa-Ivaldi, F. A., and Giszter, S. F. 1992. Does the nervous system use equilibrium-point control to guide single and multiple joint movements? Behavorial and Brain Sciences 15(4):603-613.

Boff, K. R. and Lincoln, J. E. 1988. Engineering Data Compendium: Human Perception and Performance, Harry G. Armstrong Aerospace Medical Research Laboratory, Wright-Patterson A. F. B., OH.

Boitier, V. 1996. Design and control of a two DoF SCARAtype robot actuated with McKibben pneumatic artificial muscles. Ph.D. Thesis, INSA de Toulouse, France.

Bouisset, S. 1987. The tension-length and force-velocity relationships of human muscle in situ. BioMechanics of Engineering Modelling, Simulation, Control, A. Morecki, editor, Springer-Verlag, Berlin, pp. 133-155.

Bridgestone Corporation. 1987. Soft Arm ACFAS Robot System. Tokyo, Japan.

Bridgestone Corporation and Taicubo Engineering. 1993. Soft Boy: Advanced Painting System Unit. Tokyo, Japan.

Cai, D. and Yamaura, H. 1997. A VSS control method for a manipulator driven by an artificial muscle actuator. Electronics and Communications in Japan, Part 3 80(3):55-63.

Caldwell, D. G., Medrano-Cerda, G. A., and Goodwin, M. 1995. Control of pneumatic muscle actuators. IEEE Control Systems Magazine 15(1):40-48.

Caldwell, D. G., Medrano-Cerda, G. A., and Bowler, C. J. 1997. Investigation of bipedal robot locomotion using pneumatic muscle actuators. Proceedings of the IEEE International Conference on Robotics and Automation, Albuquerque, NM, pp. 799-804.

Caldwell, D. G., Tsagarakis, N., Artrit, P., and MedranoCerda, G. A. 1999. Bio-mimetic principles in actuator design for a humanoid robot. European Journal of Mechanical and Environmental Engineering 44(2):75-80.

Chou, C-P. and Hannaford, B. 1996. Measurement and modeling of McKibben pneumatic artificial muscles. IEEE Transactions on Robotics and Automation 12(1):90-102.

Davis, S., Tsagarakis, N., Canderle, J., and Caldwell, D. G. 2003. Enhanced modeling and performance in braided pneumatic muscle actuators. International Journal of Robotics Research 22(3-4):213-227.
E.P.W. 1984. Rubber muscles take robotics one step further. Rubber Development 37(4):117-119.

Ghez,C. 1991. Muscles: effectors of the motor system. Principles of Neural Science, E. R. Kandel, J. H. Schwartz, and T. M. Jessekk, editors, Prentice-Hall, Englewood Cliffs, NJ, pp. 548-563.

Hajian, A. Z., Sanchez, D. S., and Howe, R. D. 1997. Drum roll: increasing bandwidth through passive impedance modulation. Proceedings of the IEEE International Conference on Robotics and Automation, Albuquerque, NM, pp. 2294-2299.

Hannaford, B., Winters, J. M, Chou, C. P., and Marbot, P. 1995. The anthroform biorobotic arm: a system for the study of spinal circuits. Annals of Biomedical Engineering 23:399-408.

Hebert, M., Kant, R., and De Gennes, P.-G. 1997. Dynamics and thermodynamics of artificial muscles based on nematic gels. Journal de Physique 7(7):909-919.

Hesselroth, T., Sarkar, K., Van der Smagt, P., and Schulten, K. 1994. Neural network control of a pneumatic robot arm. IEEE Transactions on Systems, Man and Cybernetics 24(1):28-37.

Hirai, K., Hirose, M., Haikawa, Y., and Takenaka, T. 1998. The development of Honda humanoid robot. Proceedings of the IEEE International Conference on Robotics and Automation, Leuwen, Belgium, pp. 1321-1326.

Hollerbach, J.M. 1985. Optimum kinematic design for a seven degrees of freedom manipulator. Proceedings of the 2nd International Symposium on Robotics Research, H. Hanafusa and H. Inoue, editors, MIT Press, Cambridge, MA, pp. 215-222.

Inoue, K. 1988. Rubbertuators and applications for robots. Proceedings of the 4th International Symposium on Robotics Research, Cambridge, MA, pp. 57-63.

Kaneko, D., Jian Ping Gong, Y., and Osada, Y. 2002. Polymer gels as soft and wet chemomechanical systems - an approach to artificial muscles. Journal of Material Chemistry 12(8):2169-2177.

Kapandji, I. A. 1982. Physiology of the Joints, Volume 1, Upper Limb, 5th edition, Churchill Livingstone, Edinburgh.

Kawamura, K., Peters, R. A. II, Baghi, S., Iskarous, M., and Bishay, M. 1995. Intelligent robotic systems in service of the disabled. IEEE Transactions on Rehabilitation Engineering 1(3):14-21.

Klute, G. K., Czerniecki, J. M., and Hannaford, B. 1999. McKibben artificial muscles: pneumatic actuators with biomechanical intelligence actuator. Proceedings of the IEEE/ASME International Conference on Advanced Intelligent Mechatronics (AIM'99), Atlanta, GA, pp. 19-22.

Klute, G. K., Czerniecki, J. M., and Hannaford, B. 2002. Artificial muscles: actuators for biorobotic systems. International Journal of Robotics Research 21(4):295-309.

Kreutz-Delgado,K.,Long, M., and Seraji,H. 1992.Kinematic analysis of 7-DOF manipulators. International Journal oI 
Robotics Research 11(5):469-481.

Matsushita, M. 1968. Synthesis of rubber artificial muscle. Journal of the Society of Instrument and Control Engineers 7(12):110-116 (in Japanese).

Martin, J. E. and Anderson, R. A. 1999. Electrostriction in field-structured composites: basis for a fast artificial muscle. Journal of Chemical Physics 111(9):4273-4281.

Noritsugu, T., Tanaka, T., and Yamanaha, T. 1996. Application of rubber artificial muscle manipulator as a rehabilitation robot. Proceedings of the IEEE International Workshop on Robot and Human Communication, Tsukuba, Japan, pp. 112-117.

Pack, R. T., Christopher, J. L. Jr., and Kawamura, K. 1997. A rubbertuator-based structure-climbing inspection robot. Proceedings of the IEEE International Conference on Robotics and Automation, Albuquerque, NM, pp. 18691874.

Rosheim, M. E. 1994. Robot Evolution. The Development of Anthrobotics. Wiley, New York.

Schulte, H.F. 1961. The characteristics of the McKibben artificial muscle. The Application of External Power in Prosthetics and Orthotics, Appendix H, Publication 874, National Academy of Sciences, Washington, DC, pp.94-115.

Seraji, H., Long, M., and Lee, T. S. 1993. Motion control of 7-DOF arms: the configuration control approach. IEEE Transactions on Robotics and Automation 9(2):125-139.

Tondu, B. and Lopez, P. 1995. Theory of an artificial pneumatic muscle and application to the modelling of McKibben artificial muscle. C.R.A.S. French National Academy of Sciences, Series IIb, 320, pp. 105-114 (in French with an abridged English version).

Tondu, B. and Lopez, P. 2000. Modeling and control of McKibben artificial muscle robot actuators. IEEE Control Systems Magazine 20(2):15-38.

Tuijhof, G. J. M. and Herder, J. L. 2000. Design, actuation and control of an anthropomorphic robot arm. Mechanism and Machine Theory 35:945-962.

Tsagarakis, N.G. and Caldwell,D. G. 2003. Development and control of a "soft-actuated" exoskeleton for use in physiotherapy and training. Autonomous Robots 15(1):21-33 (special issue on rehabilitation robotics).

Van der Smagt, P., Groen, F., and Schulten, K. 1996. Analysis and control of a rubbertuator arm. Biological Cybernetics 75:433-440.

Winter, D. A. 1969. Anthropometry (Chapter 3) and Anthropometry, Kinematic and Force Plate Data (Appendix A). Biomechanics of Human Movement. Wiley, New Yo 